\title{
PARTIAL REGULARITY RESULTS FOR MINIMIZERS OF QUASICONVEX FUNCTIONALS OF HIGHER ORDER
}

\author{
Manfred KRONZ \\ Mathematisches Institut der Friedrich-Alexander-Universität, \\ Erlangen-Nürnberg, Bismarckstr. 1 1/2, D-91054 Erlangen, Germany
}

Received 13 October 2000, revised 5 December 2000

ABSTRACT. - We consider minimizers $u \in W^{m, p}\left(\Omega, \mathbb{R}^{N}\right)$ of uniformly strictly quasiconvex functionals $F(u)=\int_{\Omega} f\left(D^{m} u\right) \mathrm{d} \mathcal{L}^{n}$ of higher order. Here $\Omega$ is a domain in $\mathbb{R}^{n}, m \geqslant 1$, and $f$ is a $C^{2}$-integrand with growth of order $p, p \geqslant 2$. Using the technique of harmonic approximation we give a direct proof of almost everywhere $C^{m, \alpha}$-regularity for such minimizers.

(C) 2002 L'Association Publications de l'Institut Henri Poincaré. Published by Elsevier B.V. All rights reserved

RÉSUMÉ. - Nous étudions des minimiseurs $u \in W^{m, p}\left(\Omega, \mathbb{R}^{N}\right)$ des fonctionnelles $F(u)=$ $\int_{\Omega} f\left(D^{m} u\right) \mathrm{d} \mathcal{L}^{n}$ uniformément strictement quasiconvexes du ordre $2 m, m \geqslant 1$. Ici, $\Omega \subset \mathbb{R}^{n}$ est une domaine et $f$ est une fonction de classe $C^{2}$ avec une crossance du ordre $p, p \geqslant 2$. À l'aide de la technique d'approximation harmonique nous obtenons une preuve directe pour la regularité de classe $C^{m, \alpha}$ pour les minimiseurs dans un ensemble ouvert $\widetilde{\Omega} \subset \Omega$ avec $\mathcal{L}^{n}(\Omega \backslash \widetilde{\Omega})=0$.

(C) 2002 L'Association Publications de l'Institut Henri Poincaré. Published by Elsevier B.V. All rights reserved

\section{Introduction}

In this paper we are interested in the question on the regularity of minimizers of quasiconvex functionals of higher order. Precisely we consider minimizers of functionals of the form

$$
F(u)=\int_{\Omega} f\left(D^{m} u\right) \mathrm{d} \mathcal{L}^{n},
$$

where $\Omega$ is a domain in $\mathbb{R}^{n}, u$ is a function in $W^{m, p}\left(\Omega, \mathbb{R}^{N}\right), p \geqslant 2, \mathcal{L}^{n}$ is the $n$ dimensional Lebesgue measure and $f$ is a strictly quasiconvex $C^{2}$-function which maps symmetric $m$-linear functions from $\mathbb{R}^{n}$ to $\mathbb{R}^{N}, m \geqslant 1$, to $\mathbb{R}$.

Considering these settings, the questions on existence and regularity of such minimizers appear. Imposing for example Dirichlet boundary conditions, i.e. $\left\{u, D u, \ldots, D^{m-1} u\right\}$ are prescribed on $\partial \Omega$, the question on existence of minimizers can be answered by proving that the functional is lower semicontinuous with respect to weak convergence in the Sobolev space $W^{m, p}\left(\Omega, \mathbb{R}^{N}\right)$. The quasiconvexity condition as a sufficient condition for 
lower semicontinuity of certain functionals in the calculus of variations was first introduced by Morrey [19]. He proved that a necessary and sufficient condition for weak lower semicontinuity in the case of certain first order functionals $F$, i.e. $m=1$, on certain Sobolev spaces is a quasiconvexity condition on the integrand $f$. That means that for all $\varphi \in C_{0}^{\infty}\left(\Omega, \mathbb{R}^{N}\right)$ and all linear functions $A: \mathbb{R}^{n} \rightarrow \mathbb{R}^{N}$

$$
\int_{\Omega} f(A) \mathrm{d} \mathcal{L}^{n} \leqslant \int_{\Omega} f(A+D \varphi) \mathrm{d} \mathcal{L}^{n} .
$$

Further results on the quasiconvexity condition on functionals $F$ were given by Meyers [18], Ball [2], Acerbi and Fusco [1], Marcellini [17], Zhou [22] and Guidorzi and Poggiolini [15]. Especially one result, going back on Meyers [18] and also proved in the last paper [15], shows that a quasiconvexity condition on the functional (1) of higher order and $p$-growth conditions

$$
0 \leqslant f(A) \leqslant L\left(1+|A|^{p}\right)
$$

for the integrand $f$ are sufficient for weak lower semicontinuity in the Sobolev space $W^{m, p}\left(\Omega, \mathbb{R}^{N}\right)$. Here a quasiconvexity condition for the integrand $f$ means that inequality (2) (with $D^{m} \varphi$ instead of $D \varphi$ ) is satisfied for all $\varphi \in C_{0}^{\infty}\left(\Omega, \mathbb{R}^{N}\right)$ and all symmetric $m$-linear $\mathbb{R}^{N}$-valued functions $A$ on $\mathbb{R}^{n}$. This growth conditions for a quasiconvex integrand $f$ together with an auxiliary coercivity condition

$$
\frac{1}{L}\left(1+|A|^{p}\right) \leqslant f(A)
$$

imply for given Dirichlet boundary data - according to the direct method in the calculus of variations - the existence of at least one minimizer $u \in W^{m, p}\left(\Omega, \mathbb{R}^{N}\right)$ of the functional $F$.

For functionals $F(u)=\int_{\Omega} f(D u) \mathrm{d} \mathcal{L}^{n}$ of first order with uniformly strictly quasiconvex integrand $f$, fulfilling the $p$-growth condition (3), partial regularity results for minimizers were derived by Evans [9]. He proved, using the indirect approach of blowing-up, that for each $0<\alpha<1$ minimizers of $F$ are locally $C^{1, \alpha}$ on an open subset $U \subset \Omega$ with $\mathcal{L}^{n}(\Omega \backslash U)=0$.

In this paper we extend the results of Evans on the partial regularity theory for minimizes of quasiconvex functionals of first order to those of minimizers of quasiconvex functionals of higher order.

To prove partial regularity results for minimizers of such functionals, we assume, that the integrand $f$ in (1) is uniformly strictly quasiconvex, which means (in the case $p=2$ )

$$
\int_{\Omega}\left(f\left(A+D^{m} \varphi\right)-f(A)\right) \mathrm{d} \mathcal{L}^{n} \geqslant \lambda \int_{\Omega}\left|D^{m} \varphi\right|^{2} \mathrm{~d} \mathcal{L}^{n}
$$

for all test functions $\varphi \in C_{0}^{\infty}\left(\Omega, \mathbb{R}^{N}\right)$ and any symmetric $m$-linear $\mathbb{R}^{N}$-valued $A$ on $\mathbb{R}^{n}$.

The following partial regularity results are proven precisely: 
THEOREM 1. - Let $u \in W^{m, 2}\left(\Omega, \mathbb{R}^{N}\right)$ be a minimizer of the functional (1) with uniformly strictly quasiconvex $C^{2}$-integrand $f$ fulfilling the growth condition $\left|D^{2} f(A)\right| \leqslant L$ for every symmetric m-linear $\mathbb{R}^{N}$-valued function $A$ on $\mathbb{R}^{n}$. Then $u$ is locally $C^{m, \alpha}$ continuous for every $0<\alpha<1$ on an open set $U$ with $\mathcal{L}^{n}(\Omega \backslash U)=0$.

THEOREM 2. - Let $u \in W^{m, p}\left(\Omega, \mathbb{R}^{N}\right), p \geqslant 2$, be a minimizer of the functional (1) with uniformly strictly quasiconvex $C^{2}$-integrand $f$ fulfilling the growth condition $D^{2} f(A) \leqslant L\left(1+|A|^{p-2}\right)$ for every symmetric m-linear $\mathbb{R}^{N}$-valued function $A$ on $\mathbb{R}^{n}$. Then $u$ is locally $C^{m, \alpha}$-continuous for every $0<\alpha<1$ on an open set $U$ with $\mathcal{L}^{n}(\Omega \backslash U)=0$.

As in the case of first order problems the uniformly strictly quasiconvexity condition for $f$ implies that the corresponding Euler equations for $F$ are elliptic in the sense of Legendre-Hadamard (see Remark 3 below). With regard to regularity theory, uniform strict quasiconvexity permits the proof of a Caccioppoly inequality - sometimes called reverse Poincaré inequality - for minimizers of $F$. After showing that a in terms of Caccioppoli's inequality rescaled minimizer is nearly a solution of a strictly elliptic constant coefficient system, we derive an excess-decay estimate on an open set $U \subset \Omega$. This is done, using the technique of $\mathcal{A}$-harmonic approximation, as expressed in Lemma 6. For harmonic approximation, this technique has its origins in Simon's proof of the regularity theorem of Allard (see [20]). The point of this approximation technique is to show that for a bilinear form $\mathcal{A}$, which is elliptic in the sense of LegendreHadamard, a function $g$ which is "approximately $(\mathcal{A}, m)$-harmonic" - that means $\int_{\Omega} \mathcal{A}\left(D^{m} g, D^{m} \varphi\right) \mathrm{d} \mathcal{L}^{n}$ is sufficiently small for all test functions $\varphi$ - lies $L^{2}$-close to some $(\mathcal{A}, \mathrm{m})$-harmonic function $v$ - that means a function $v$ with $\int_{\Omega} \mathcal{A}\left(D^{m} v, D^{m} \varphi\right) \mathrm{d} \mathcal{L}^{n}=0$ for all test functions $\varphi$. Then, standard a priori estimates for solutions of elliptic constant coefficient systems can be used to derive estimates for excess terms. Precisely we deal with $\mathcal{A}=D^{2} f\left(D^{m} P_{x_{0}, \rho}\right)$ where $P_{x_{0}, \rho}$ is a polynomial of degree at most $m$, associated with the minimizer $u$ of the functional $F$ on a ball $B_{\rho}\left(x_{0}\right)$. Using the $(\mathcal{A}, m)$-harmonic approximation technique we derive decay estimates which can be iterated for our excess term (we restrict here to the case $p=2$ )

$$
\Psi_{2}\left(x_{0}, \rho, P_{x_{0}, \rho}\right)=\left(\rho^{-2} f_{B_{\rho}\left(x_{0}\right)}\left|D^{m-1}\left(u-P_{x_{0}, \rho}\right)\right|^{2} \mathrm{~d} \mathcal{L}^{n}\right)^{1 / 2}
$$

in points $x_{0} \in \Omega$, where $\Psi_{2}\left(x_{0}, \rho, P_{x_{0}, \rho}\right)$ is sufficiently small. The smallness of this quantity can be ensured on an open set $U$ of full Lebesgue measure in $\Omega$. Iterating this excess term, we derive estimates for $\int_{B_{\rho}\left(x_{0}\right)}\left|u-P_{x_{0}, \rho}\right|^{2} \mathrm{~d} \mathcal{L}^{n}$ from which we infer, applying an integral characterization for $C^{m, \alpha}$-functions due to Campanato ([5], [6]), local $C^{m, \alpha}$-regularity of minimizers $u$ of $F$ on the open set $U$.

Beginning with notations and preliminary lemmas (special kinds of Poincaré's and Sobolev's inequalities, remarks on polynomials, minimizing the $L^{2}$-distance to given $L^{2}$-maps) in Section 2, the main results Theorem 1 and Theorem 2 of this paper are formulated in Section 3. After proving the above mentioned Caccioppoli inequality in Section 4, we study the - for our settings - suitable harmonic approximation technique in Section 5. Finally, we give proofs of our theorems in the last two sections. 
At last we should mention the following remarks on our proofs: The $(\mathcal{A}, m)$-harmonic approximation lemma is the only time where we argue indirectly. In all the other parts of this paper we argue directly. This leads to explicit constants in our estimates and some quantitative control of the smallness conditions. Furthermore, even in the case $p>2$ of non-quadratic growth of the integrand $f$ in functional (1) we only use $L^{2}$-theory, i.e. standard a priori estimates for solutions of elliptic constant coefficient systems, to derive partial regularity for minimizers of non-quadratic functionals.

\section{Notations and preliminary lemmas}

As mentioned above, we consider a domain $\Omega$ in $\mathbb{R}^{n}$ and mappings from $\Omega$ to $\mathbb{R}^{N}$ with $n \geqslant 2$ and $N \geqslant 1$. We write $\mathcal{L}^{n}$ for the $n$-dimensional Lebesgue measure. For a $L^{1}$-function $u: \Omega \rightarrow \mathbb{R}^{N}$ we denote the average of $u$ over a ball $B_{\rho}\left(x_{0}\right) \subset \Omega$ by

$$
u_{x_{0}, \rho}=f_{B_{\rho}\left(x_{0}\right)} u \mathrm{~d} \mathcal{L}^{n}=\frac{1}{\mathcal{L}^{n}\left(B_{\rho}\left(x_{0}\right)\right)} \int_{B_{\rho}\left(x_{0}\right)} u \mathrm{~d} \mathcal{L}^{n} .
$$

$\bigodot^{k}\left(\mathbb{R}^{n}, \mathbb{R}^{N}\right)$ will be the vectorspace of symmetric $k$-linear $\mathbb{R}^{N}$-valued functions on $\mathbb{R}^{n}$. Since the dimension of this vectorspace is $N\left(\begin{array}{c}n+k-1 \\ k\end{array}\right)$ we may identify this space with the

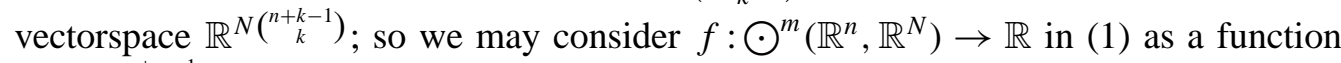

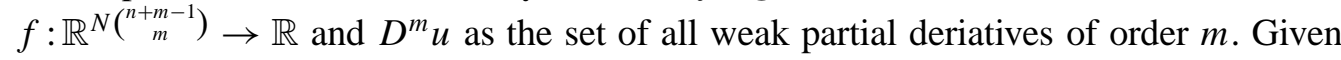
$\zeta \in \mathbb{R}^{n}$ the tensor product

$$
\underbrace{\zeta \otimes \cdots \otimes \zeta}_{m \text { times }}=: \zeta^{m}
$$

is symmetric and $m$-linear and hence an element of $\bigodot^{m}\left(\mathbb{R}^{n}, \mathbb{R}\right)$. So, for $\eta \in \mathbb{R}^{N}$, the tensor product $\zeta^{m} \otimes \eta$ is an element of $\odot^{m}\left(\mathbb{R}^{n}, \mathbb{R}^{N}\right)$.

Here, we first prove a Poincaré inequality which is suitable to our setting:

LEMMA 1. - Let $u, v \in W^{m, p}\left(B_{\rho}\left(x_{0}\right), \mathbb{R}^{N}\right)$ with $\int_{B_{\rho}\left(x_{0}\right)} D^{k}(u-v) \mathrm{d} \mathcal{L}^{n}=0$ for all $0 \leqslant k \leqslant l-1(\leqslant m-1)$. Then there exists a constant $C_{P}=C_{P}(n, N, l)$ such that

$$
\left(\int_{B_{\rho}\left(x_{0}\right)}\left|D^{k}(u-v)\right|^{p} \mathrm{~d} \mathcal{L}^{n}\right)^{1 / p} \leqslant C_{P} \rho^{l-k}\left(\int_{B_{\rho}\left(x_{0}\right)}\left|D^{l}(u-v)\right|^{p} \mathrm{~d} \mathcal{L}^{n}\right)^{1 / p} .
$$

Proof. - Let $w^{k}=D^{k}(u-v)$ with $w^{k} \in W^{m-k, p}\left(\Omega, \bigodot^{k}\left(\mathbb{R}^{n}, \mathbb{R}^{N}\right)\right)$ and $w_{x_{0}, \rho}^{k}=0$. Then the ususal Poincaré inequality shows:

$$
\begin{aligned}
\left(\int_{B_{\rho}\left(x_{0}\right)}\left|D^{k}(u-v)\right|^{p} \mathrm{~d} \mathcal{L}^{n}\right)^{1 / p} & =\left(\int_{B_{\rho}\left(x_{0}\right)}\left|w^{k}-w_{x_{0}, \rho}^{k}\right|^{p} \mathrm{~d} \mathcal{L}^{n}\right)^{1 / p} \\
& \leqslant C_{k}(n, N) \rho\left(\int_{B_{\rho}\left(x_{0}\right)}\left|D w^{k}\right|^{p} \mathrm{~d} \mathcal{L}^{n}\right)^{1 / p}
\end{aligned}
$$




$$
\begin{aligned}
& =C_{k}(n, N) \rho\left(\int_{B_{\rho}\left(x_{0}\right)}\left|D^{k+1}(u-v)\right|^{p} \mathrm{~d} \mathcal{L}^{n}\right)^{1 / p} \leqslant \cdots \\
& \leqslant \prod_{j=k}^{l-1} C_{j}(n, N) \rho^{l-k}\left(\int_{B_{\rho}\left(x_{0}\right)}\left|D^{l}(u-v)\right|^{p} \mathrm{~d} \mathcal{L}^{n}\right)^{1 / p} \\
& =C_{P}(n, N, l) \rho^{l-k}\left(\int_{B_{\rho}\left(x_{0}\right)}\left|D^{l}(u-v)\right|^{p} \mathrm{~d} \mathcal{L}^{n}\right)^{1 / p} .
\end{aligned}
$$

Note that the constants $C_{j}(n, N)$ depend on the dimension of $\Omega$ and the dimension of the vectorspaces $\bigodot^{j}\left(\mathbb{R}^{n}, \mathbb{R}^{N}\right)$.

A fruitful way, showing that a given $L^{p}$-function has a Hölder continuous representative is Campanato's integral characterization of Hölder continuous functions (see [12, Theorem 3.1]). Campanato has also derived an integral characterization for $C^{m, \alpha}$ functions [3,4], from which it follows, that a function $v \in L^{p}\left(\Omega, \mathbb{R}^{N}\right)$ is $C^{m, \alpha}$ near $x_{0} \in \Omega$, $0<\alpha \leqslant 1,1<p<\infty$, if there exists a constant $C$ independent on $\rho$ and $x_{0}$ such that

$$
\left(f_{B_{r}(y)}\left|u-P_{y, r}\right|^{p} \mathrm{~d} \mathcal{L}^{n}\right)^{1 / p} \leqslant C r^{m+\alpha}
$$

for all $y$ near $x_{0}$ and all $r \leqslant \rho$. Here $P_{y, r}$ is the unique polynomial of degree $\leqslant m$ minimizing

$$
P \mapsto \int_{B_{r}(y)}|u-P|^{p} \mathrm{~d} \mathcal{L}^{n}
$$

among all polynomials $P: B_{r}(y) \rightarrow \mathbb{R}^{N}$ of degree $\leqslant m$. We will use this result in the proof of our main theorems. We are also dealing with the explicit structure of the polynomial $P_{x_{0}, \rho}$ of degree 1 minimizing

$$
P \mapsto f_{B_{\rho}\left(x_{0}\right)}|u-P|^{2} \mathrm{~d} \mathcal{L}^{n}:=\Psi(P),
$$

$u \in L^{2}\left(B_{\rho}\left(x_{0}\right), \mathbb{R}^{N}\right)$ given, among all polynomials $P: B_{\rho}\left(x_{0}\right) \rightarrow \mathbb{R}^{N}$ of degree $\leqslant 1$. To get an explicit formula for $P_{x_{0}, \rho}$ we note first, that $\Psi$ is a strict convex, coercive and continuous on the finite dimensional space of polynomials of degree $\leqslant 1$. Therefore a unique minimum point $P_{x_{0}, \rho}$ of $\Psi$ exists. We write

$$
P_{x_{0}, \rho}(x)=q_{x_{0}, \rho}+Q_{x_{0}, \rho}\left(x-x_{0}\right) .
$$

A straightforward elementary computation gives that

$$
q_{x_{0}, \rho}=f_{B_{\rho}\left(x_{0}\right)} u \mathrm{~d} \mathcal{L}^{n}=u_{x_{0}, \rho}
$$


is the mean value of $u$ on $B_{\rho}\left(x_{0}\right)$ and

$$
Q_{x_{0}, \rho}=\frac{n+2}{\rho^{2}} f_{B_{\rho}\left(x_{0}\right)} u(x) \otimes\left(x-x_{0}\right) \mathrm{d} \mathcal{L}^{n}(x)
$$

is a momentum.

Since $\int_{B_{\rho}\left(x_{0}\right)}\left(u-P_{x_{0}, \rho}\right) \mathrm{d} \mathcal{L}^{n}=0$, we get a Poincaré inequality for $u \in W^{1, p}\left(\Omega, \mathbb{R}^{N}\right)$ of the following form:

$$
\left(\int_{B_{\rho}\left(x_{0}\right)}\left|u-P_{x_{0}, \rho}\right|^{p} \mathrm{~d} \mathcal{L}^{n}\right)^{1 / p} \leqslant C_{p} \rho\left(\int_{B_{\rho}\left(x_{0}\right)}\left|D\left(u-P_{x_{0}, \rho}\right)\right|^{p} \mathrm{~d} \mathcal{L}^{n}\right)^{1 / p} .
$$

In an analogous way we get Sobolev inequalities for $u \in W^{1, p}\left(\Omega, \mathbb{R}^{N}\right)$. If

$$
p^{*}= \begin{cases}\frac{n p}{n-p} & \text { if } 1 \leqslant p<n, \\ p^{*} \in[1, \infty[\text { fixed } & \text { if } p \geqslant n,\end{cases}
$$

the usual Sobolev inequality for $u \in W^{1, p}\left(\Omega, \mathbb{R}^{N}\right)$ states: $^{1}$

$$
\left(f_{B_{\rho}\left(x_{0}\right)}\left|u-u_{x_{0}, \rho}\right|^{p^{*}} \mathrm{~d} \mathcal{L}^{n}\right)^{1 / p^{*}} \leqslant C_{S} \rho\left(f_{B_{\rho}\left(x_{0}\right)}|D u|^{p} \mathrm{~d} \mathcal{L}^{n}\right)^{1 / p} .
$$

This inequality implies

$$
\left(f_{B_{\rho}\left(x_{0}\right)}\left|u-P_{x_{0}, \rho}\right|^{p^{*}} \mathrm{~d} \mathcal{L}^{n}\right)^{1 / p^{*}} \leqslant C_{S} \rho\left(f_{B_{\rho}\left(x_{0}\right)}\left|D\left(u-P_{x_{0}, \rho}\right)\right|^{p} \mathrm{~d} \mathcal{L}^{n}\right)^{1 / p} .
$$

LEMMA 2. - Let $u \in L^{2}\left(B_{\rho}\left(x_{0}\right)\right), 0<\theta \leqslant 1, q_{x_{0}, \theta \rho}, q_{x_{0}, \rho}$ as in (5), $Q_{x_{0}, \theta \rho}, Q_{x_{0}, \rho}$ as in (6) and $P_{x_{0}, \rho}$ the polynomial of degree 1 with $P_{x_{0}, \rho}(x)=q_{x_{0}, \rho}+Q_{x_{0}, \rho}\left(x-x_{0}\right)$. Then the following estimates hold:

$$
\begin{gathered}
\text { (i) }\left|Q_{x_{0}, \theta \rho}-Q_{x_{0}, \rho}\right|^{2} \leqslant n(n+2)(\theta \rho)^{-2} \underset{B_{\theta \rho}\left(x_{0}\right)}{f}\left|u-P_{x_{0}, \rho}\right|^{2} \mathrm{~d} \mathcal{L}^{n} ; \\
\text { (ii) }\left|D P_{x_{0}, \rho}-(D u)_{x_{0}, \rho}\right|^{2} \leqslant C_{p}^{2} n(n+2) f_{B_{\rho}\left(x_{0}\right)}\left|D u-(D u)_{x_{0}, \rho}\right|^{2} \mathrm{~d} \mathcal{L}^{n} .
\end{gathered}
$$

Proof. - For (i), first we observe that for every linear map $Q$ from $\mathbb{R}^{n}$ to $\mathbb{R}^{N}$

$$
\int_{B_{\theta \rho}\left(x_{0}\right)} Q\left(x-x_{0}\right) \otimes\left(x-x_{0}\right) \mathrm{d} \mathcal{L}^{n}(x)=\frac{(\theta \rho)^{2}}{n+2} Q
$$

\footnotetext{
${ }^{1}$ For $p<n$ see [10, p. 141]; for $p>n$ see Morrey's inequality in [10, p. 143]; for the critical case $p=n$ the inequality follows from $B M O$-theory, for example from [16, Theorem 7.21]; in the case $p \leqslant n$ the constant $C_{s}$ depends on $p^{*}$.
} 
and for every constant $q \in \mathbb{R}^{N}$

$$
f_{B_{\theta \rho}\left(x_{0}\right)} q \otimes\left(x-x_{0}\right) \mathrm{d} \mathcal{L}^{n}(x)=0 .
$$

Therefore we can compute

$$
\begin{aligned}
& \left|Q_{x_{0}, \theta \rho}-Q_{x_{0}, \rho}\right|^{2} \\
& \quad=\left(\frac{n+2}{(\theta \rho)^{2}}\right)^{2}\left|f_{B_{\theta \rho}\left(x_{0}\right)}\left(u-q_{x_{0}, \rho}-Q_{x_{0}, \rho}\left(x-x_{0}\right)\right) \otimes\left(x-x_{0}\right) \mathrm{d} \mathcal{L}^{n}(x)\right|^{2} \\
& \quad \leqslant \frac{(n+2)^{2}}{(\theta \rho)^{4}} f_{B_{\theta \rho}\left(x_{0}\right)}\left|u-q_{x_{0}, \rho}-Q_{x_{0}, \rho}\left(x-x_{0}\right)\right|^{2} \mathrm{~d} \mathcal{L}^{n}(x) \underset{B_{\theta \rho}\left(x_{0}\right)}{f}\left|x-x_{0}\right|^{2} \mathrm{~d} \mathcal{L}^{n}(x) \\
& \quad=n(n+2)(\theta \rho)^{-2} \underset{B_{\theta \rho}\left(x_{0}\right)}{f}\left|u-P_{x_{0}, \rho}\right|^{2} \mathrm{~d} \mathcal{L}^{n} .
\end{aligned}
$$

To prove (ii), we see, using Poincaré's inequality,

$$
\begin{aligned}
& \left|D P_{x_{0}, \rho}-(D u)_{x_{0}, \rho}\right|^{2} \\
& =\left(\frac{n+2}{\rho^{2}}\right)^{2}\left|f_{B_{\rho}\left(x_{0}\right)}\left(u-u_{x_{0}, \rho}-(D u)_{x_{0}, \rho}\left(x-x_{0}\right)\right) \otimes\left(x-x_{0}\right) \mathrm{d} \mathcal{L}^{n}(x)\right|^{2} \\
& \quad \leqslant n(n+2) \rho^{-2} f_{B_{\rho}\left(x_{0}\right)}\left|u-u_{x_{0}, \rho}-(D u)_{x_{0}, \rho}\left(x-x_{0}\right)\right|^{2} \mathrm{~d} \mathcal{L}^{n} . \\
& \quad \leqslant C_{p}^{2} n(n+2) f_{B_{\rho}\left(x_{0}\right)}\left|D u-(D u)_{x_{0}, \rho}\right|^{2} \mathrm{~d} \mathcal{L}^{n} .
\end{aligned}
$$

\section{Hypotheses and statements of results}

Assume that the functional (1) fulfills the following conditions:

\section{Case $p=2$}

(H1) The $C^{2}$-function $f$ is (uniformly) strictly quasiconvex, i.e. there exists a constant $\lambda>0$ such that for all balls $B_{\rho}\left(x_{0}\right) \Subset \Omega$, all $A \in \bigodot^{m}\left(\mathbb{R}^{n}, \mathbb{R}^{N}\right)$ and all $\varphi \in C_{0}^{\infty}\left(B_{\rho}\left(x_{0}\right), \mathbb{R}^{N}\right)$ there holds

$$
\int_{B_{\rho}\left(x_{0}\right)}\left(f\left(A+D^{m} \varphi\right)-f(A)\right) \mathrm{d} \mathcal{L}^{n} \geqslant \lambda \int_{B_{\rho}\left(x_{0}\right)}\left|D^{m} \varphi\right|^{2} \mathrm{~d} \mathcal{L}^{n}
$$

(H2) there exists $L \geqslant 0$ such that for all $A \in \bigodot^{m}\left(\mathbb{R}^{n}, \mathbb{R}^{N}\right) D^{2} f$ is uniformly continuous with

$$
\left|D^{2} f(A)\right| \leqslant L
$$


The condition that $D^{2} f$ in (H2) is uniformly continuous, can be removed, see Remark 4 below and hypothesis (H4).

\section{Case $p \geqslant 2$}

(H3) The $C^{2}$-function $f$ is (uniformly) strictly quasiconvex, i.e. there exists a constant $\lambda>0$ such that for all balls $B_{\rho}\left(x_{0}\right) \Subset \Omega$, all $A \in \odot^{m}\left(\mathbb{R}^{n}, \mathbb{R}^{N}\right)$ and all $\varphi \in C_{0}^{\infty}\left(B_{\rho}\left(x_{0}\right), \mathbb{R}^{N}\right)$ there holds

$$
\int_{B_{\rho}\left(x_{0}\right)}\left(f\left(A+D^{m} \varphi\right)-f(A)\right) \mathrm{d} \mathcal{L}^{n} \geqslant \lambda \int_{B_{\rho}\left(x_{0}\right)}\left(\left|D^{m} \varphi\right|^{2}+\left|D^{m} \varphi\right|^{p}\right) \mathrm{d} \mathcal{L}^{n} ;
$$

(H4) there exists $L \geqslant 0$ such that for all $A \in \odot^{m}\left(\mathbb{R}^{n}, \mathbb{R}^{N}\right)$ there holds

$$
\left|D^{2} f(A)\right| \leqslant L\left(1+|A|^{p-2}\right) .
$$

Remark 1. - Notice that the conditions (H2) resp. (H4) imply

$$
\begin{gathered}
|f(A)| \leqslant \operatorname{Const}\left(1+|A|^{2}\right), \quad|D f(A)| \leqslant \operatorname{Const}(1+|A|), \\
\text { resp. }|f(A)| \leqslant \operatorname{Const}\left(1+|A|^{p}\right), \quad|D f(A)| \leqslant \operatorname{Const}\left(1+|A|^{p-1}\right) .
\end{gathered}
$$

From this we infer

- that (H1) respectively (H3) is valid for all $\varphi \in W_{0}^{m, 2}\left(B_{\rho}\left(x_{0}\right), \mathbb{R}^{N}\right)$ respectively for all $\varphi \in W_{0}^{m, p}\left(B_{\rho}\left(x_{0}\right), \mathbb{R}^{N}\right)$;

- that the functional $F(u)=\int_{\Omega} f\left(D^{m} u\right) \mathrm{d} \mathcal{L}^{n}$ is lower semicontinuous with respect to weak convergence in $W_{0}^{m, 2}\left(\Omega, \mathbb{R}^{N}\right)$ resp. $W_{0}^{m, p}\left(\Omega, \mathbb{R}^{N}\right)$ (see $\left.[18,15]\right)$.

Remark 2. - The Euler equations for minimizers of the functional

$$
F(u)=\int_{\Omega} f\left(D^{m} u\right) \mathrm{d} \mathcal{L}^{n}
$$

are given by (see [11, p. 14] and note that $f$ only depends on $m$-order deriviatives of $u$ ):

$$
\int_{\Omega} D f\left(D^{m} u\right) D^{m} \varphi \mathrm{d} \mathcal{L}^{n}=0
$$

for all $\varphi \in C_{0}^{\infty}\left(\Omega, \mathbb{R}^{N}\right)$.

Remark 3. - In view of Remark 1 we see that in the case (H1) for given $v \in$ $W_{0}^{m, 2}\left(\Omega, \mathbb{R}^{N}\right)$ the nonnegative function

$$
I(t)=\int_{\Omega}\left[f\left(A+t D^{m} v\right)-f(A)-\lambda t^{2}\left|D^{m} v\right|^{2}\right] \mathrm{d} \mathcal{L}^{n}
$$


attains its absolut minimum at $t=0$, hence

$$
\frac{\mathrm{d}^{2} I}{\mathrm{~d} t^{2}}(0)=\int_{\Omega}\left[D^{2} f(A)\left(D^{m} v, D^{m} v\right)-2 \lambda\left|D^{m} v\right|^{2}\right] \mathrm{d} \mathcal{L}^{n} \geqslant 0
$$

for every $v \in W_{0}^{m, 2}\left(\Omega, \mathbb{R}^{N}\right)$. In the case (H3) we obtain the same inequality for every $v \in W_{0}^{m, p}\left(\Omega, \mathbb{R}^{N}\right)$ since higher order terms vanish when carrying out the differentiation similar to (11). We have demonstrated here, that the quasiconvexity conditions (H1) resp. (H3) together with the growth conditions (H2) resp. (H4) imply

$$
\int_{\Omega} D^{2} f(A)\left(D^{m} v, D^{m} v\right) \mathrm{d} \mathcal{L}^{n} \geqslant 2 \lambda \int_{\Omega}\left|D^{m} v\right|^{2} \mathrm{~d} \mathcal{L}^{n}
$$

for all $A \in \bigodot^{m}\left(\mathbb{R}^{n}, \mathbb{R}^{N}\right)$ and for all $v \in W_{0}^{m, 2}\left(\Omega, \mathbb{R}^{N}\right)$ resp. $v \in W_{0}^{m, p}\left(\Omega, \mathbb{R}^{N}\right)$. Note that this integral condition - as in the case $m=1$ - is equivalent to the Legendre-Hadamard condition

$$
D^{2} f(A)\left(\zeta^{m} \otimes \eta, \zeta^{m} \otimes \eta\right) \geqslant \frac{2 \lambda}{m !}|\zeta|^{2 m}|\eta|^{2}
$$

for all $A \in \bigodot^{m}\left(\mathbb{R}^{n}, \mathbb{R}^{N}\right), \zeta \in \mathbb{R}^{n}$ and $\eta \in \mathbb{R}^{N}$, see [18, Theorem 7].

Remark 4. - Hypothesis (H2) - i.e. the condition that $D^{2} f$ is uniformly continuous - leads to the existence of a monotone increasing and concave function $v: \mathbb{R}_{\geqslant 0} \rightarrow \mathbb{R}_{\geqslant 0}$ with $v(0)=0$ and

$$
\left|D^{2} f(A)-D^{2} f(B)\right| \leqslant v(|A-B|)
$$

for all $A, B \in \bigodot^{m}\left(\mathbb{R}^{n}, \mathbb{R}^{N}\right)$. In the case of hypothesis (H4) there exists - see (H4) in [14, p. 194] - a continuous, non-negative function $v(t, s)$, for fixed $s$ monotone increasing in $t$ and vice versa, concave in $s$ with $v(s, 0)=0$ and

$$
\left|D^{2} f(A)-D^{2} f(B)\right| \leqslant\left(1+|A|^{p-2}+|B|^{p-2}\right) v(|A|,|A-B|)
$$

for all $A, B \in \bigodot^{m}\left(\mathbb{R}^{n}, \mathbb{R}^{N}\right)$. This implies that there exists a monotone increasing and concave function $v_{M}: \mathbb{R}_{\geqslant 0} \rightarrow \mathbb{R}_{\geqslant 0}$, with $v_{M}(0)=0$ and

$$
\left|D^{2} f(A)-D^{2} f(B)\right| \leqslant\left(1+M^{p-2}+|B|^{p-2}\right) v_{M}(|A-B|)
$$

for all $A, B \in \bigodot^{m}\left(\mathbb{R}^{n}, \mathbb{R}^{N}\right)$ with $|A| \leqslant M$. Removing the condition that $D^{2} f$ is uniformly continuous in (H2), we would get an analogous expression in the case $p=2$. This would make the arguments in the proof of Theorem 1 below a bit more complicated. Note, that the hypotheses $(\mathrm{H} 1)$ and $(\mathrm{H} 2)$ are included in $(\mathrm{H} 3)$ and $(\mathrm{H} 4)$ and therefore the more general case is included in Theorem 2 below.

We call a map $u \in W^{m, p}\left(\Omega, \mathbb{R}^{N}\right)$ a minmizer of the functional $F$ in (1) if and only if

$$
F(u) \leqslant F(u+\varphi)
$$

for every $\varphi \in W_{0}^{m, p}\left(\Omega, \mathbb{R}^{N}\right)$. 
We are now in the position to state our main results

THEOREM 1. - Suppose the functional $F$ in (1) satisfies (H1) and (H2) and $u \in$ $W^{m, 2}\left(\Omega, \mathbb{R}^{N}\right)$ is a minimizer of $F$. Then there exists an open subset $U \subset \Omega$ with

$$
\mathcal{L}^{n}(\Omega \backslash U)=0
$$

and $u \in C_{\text {loc }}^{m, \alpha}\left(U, \mathbb{R}^{N}\right)$ for every $0<\alpha<1$. Furthermore

$$
\Omega \backslash U \subset \Sigma=\left\{x_{0} \in \Omega: \liminf _{\rho \searrow 0} f_{B_{\rho}\left(x_{0}\right)}\left|D^{m} u-\left(D^{m} u\right)_{x_{0}, \rho}\right|^{2} \mathrm{~d} \mathcal{L}^{n}>0\right\} .
$$

THEOREM 2. - Suppose the functional $F$ in (1) satisfies (H3) and (H4) and $u \in$ $W^{m, p}\left(\Omega, \mathbb{R}^{N}\right)$ is a minimizer of $F$. Then there exists an open subset $U \subset \Omega$ with

$$
\mathcal{L}^{n}(\Omega \backslash U)=0
$$

and $u \in C_{l o c}^{m, \alpha}\left(U, \mathbb{R}^{N}\right)$ for every $0<\alpha<1$. Further $\Omega \backslash U \subset \Sigma_{1} \cup \Sigma_{2}$ where

$$
\begin{aligned}
& \Sigma_{1}=\left\{x_{0} \in \Omega: \liminf _{\rho \searrow 0} f_{B_{\rho}\left(x_{0}\right)}\left|D^{m} u-\left(D^{m} u\right)_{x_{0}, \rho}\right|^{p} \mathrm{~d} \mathcal{L}^{n}>0\right\}, \\
& \Sigma_{2}=\left\{x_{0} \in \Omega: \liminf _{\rho \searrow 0}\left|\left(D^{m} u\right)_{x_{0}, \rho}\right|=\infty\right\} .
\end{aligned}
$$

It is obvious that the same results hold for local minimizers of the functional $F$ in (1).

\section{Caccioppoli inequality}

In this section we prove Caccioppoli inequalities for minimizers $u \in W^{m, 2}\left(\Omega, \mathbb{R}^{N}\right)$ resp. $u \in W^{m, p}\left(\Omega, \mathbb{R}^{N}\right)$ of the functional $\int_{\Omega} f\left(D^{m} u\right) \mathrm{d} \mathcal{L}^{n}$ where $f$ satisfies (H1) and (H2) resp. (H3) and (H4). In the situation $m=1$ we refer to [9]. We start with the case $p=2$.

LEMMA 3. - Let $u \in W^{m, 2}\left(\Omega, \mathbb{R}^{N}\right)$ be a minimizer of the functional $\int_{\Omega} f\left(D^{m} u\right) \mathrm{d} \mathcal{L}^{n}$, where $f$ satisfies $(\mathrm{H} 1)$ and $(\mathrm{H} 2)$. Then there exists a constant $\widetilde{C}_{c a c}=\widetilde{C}_{c a c}(m, L, \lambda)$ such that

$$
\int_{B_{\rho / 2}\left(x_{0}\right)}\left|D^{m}(u-P)\right|^{2} \mathrm{~d} \mathcal{L}^{n} \leqslant \widetilde{C}_{c a c}^{2} \sum_{k=0}^{m-1} \rho^{2(k-m)} \int_{B_{\rho}\left(x_{0}\right)}\left|D^{k}(u-P)\right|^{2} \mathrm{~d} \mathcal{L}^{n}
$$

for all balls $B_{\rho}\left(x_{0}\right) \Subset \Omega$ and all polynomials $P: \mathbb{R}^{N} \supset \Omega \rightarrow \mathbb{R}^{N}$ of degree $\leqslant m$. Moreover, if $\int_{B_{\rho}\left(x_{0}\right)} D^{k}(u-P) \mathrm{d} \mathcal{L}^{n}=0$ for $0 \leqslant k \leqslant m-2$, then there exists $C_{\text {cac }}=$ $C_{c a c}(m, L, \lambda, n, N)$ such that

$$
\int_{B_{\rho / 2}\left(x_{0}\right)}\left|D^{m}(u-P)\right|^{2} \mathrm{~d} \mathcal{L}^{n} \leqslant C_{c a c}^{2} \rho^{-2} \int_{B_{\rho}\left(x_{0}\right)}\left|D^{m-1}(u-P)\right|^{2} \mathrm{~d} \mathcal{L}^{n} .
$$


Proof. - Take a polynomial $P$ of degree at most $m$ with $D^{m} P=: A \in \bigodot^{m}\left(\mathbb{R}^{n}, \mathbb{R}^{N}\right)$ and choose the following functions

$$
\varphi=\eta(u-P)=: \eta v, \quad \psi=(1-\eta) v,
$$

where $\left.\eta \in C_{0}^{\infty}\left(B_{\rho}\left(x_{0}\right)\right)\right)$ satisfies $(0<t<s \leqslant \rho) 0 \leq \eta \leqslant 1, \eta \equiv 1$ on $B_{t}\left(x_{0}\right), \eta \equiv 0$ on $\Omega \backslash B_{s}\left(x_{0}\right),\left|D^{k} \eta\right| \leqslant\left(C_{\eta}(s-t)\right)^{-k}$ for all $0 \leqslant k \leqslant m$. According to Remark $1, \varphi$ is admissible in (H1) with

$$
D^{m} \varphi+D^{m} \psi=D^{m} v=D^{m}(u-P)=D^{m} u-A .
$$

Using in turn (H1), (13), the minimal property of $u$ and (H2), we deduce:

$$
\begin{aligned}
\lambda \int_{B_{s}\left(x_{0}\right)}\left|D^{m} \varphi\right|^{2} \mathrm{~d} \mathcal{L}^{n} \leqslant & \int_{B_{s}\left(x_{0}\right)}\left[f\left(A+D^{m} \varphi\right)-f(A)\right] \mathrm{d} \mathcal{L}^{n} \\
= & \int_{B_{s}\left(x_{0}\right)}\left[f\left(D^{m} u-D^{m} \psi\right)-f(A)\right] \mathrm{d} \mathcal{L}^{n} \\
\leqslant & \int_{B_{s}\left(x_{0}\right)}\left[f\left(D^{m} u-D^{m} \psi\right)-f\left(D^{m} u\right)\right] \mathrm{d} \mathcal{L}^{n} \\
& +\int_{B_{s}\left(x_{0}\right)}\left[f\left(D^{m} u\right)-f\left(D^{m} u-D^{m} \varphi\right)\right] \mathrm{d} \mathcal{L}^{n} \\
& +\int_{B_{s}\left(x_{0}\right)}\left[f\left(D^{m} u-D^{m} \varphi\right)-f(A)\right] \mathrm{d} \mathcal{L}^{n} \\
\leqslant & \int_{B_{s}\left(x_{0}\right)}\left[f\left(D^{m} u-D^{m} \psi\right)-f\left(D^{m} u\right)\right] \mathrm{d} \mathcal{L}^{n} \\
& +\int_{B_{s}\left(x_{0}\right)}\left[f\left(A+D^{m} \psi\right)-f(A)\right] \mathrm{d} \mathcal{L}^{n} \\
= & \int_{B_{s}\left(x_{0}\right)} \int_{0}^{1}\left[D f\left(A+t D^{m} \psi\right)-D f\left(D^{m} u-t D^{m} \psi\right)\right] \mathrm{d} t D^{m} \psi \mathrm{d} \mathcal{L}^{n} \\
\leqslant & \int_{B_{s}\left(x_{0}\right)}\left(\left|D^{m} v\right|+\left|D^{m} \psi\right|\right)\left|D^{m} \psi\right| \mathrm{d} \mathcal{L}^{n} \\
= & L \int_{B_{s}\left(x_{0}\right) \backslash B_{t}\left(x_{0}\right)}\left(\left|D^{m} v\right|+\left|D^{m} \psi\right|\right)\left|D^{m} \psi\right| \mathrm{d} \mathcal{L}^{n} \\
\leqslant & \left.\frac{L}{2} \int_{B_{s}\left(x_{0}\right) \backslash B_{t}\left(x_{0}\right)}\left|D^{m} v\right|^{2} \mathrm{~d} \mathcal{L}^{n}+\frac{3}{2} L x_{0}\right) \\
& \left.D^{m} \psi\right|^{2} \mathrm{~d} \mathcal{L}^{n} . \\
&
\end{aligned}
$$


Noting $^{2} D^{m} \psi=D^{m}((1-\eta) v)=\sum_{k=0}^{m}\left(\begin{array}{c}m \\ k\end{array}\right) D^{m-k}(1-\eta) \otimes D^{k} v$, we proceed

$$
\begin{aligned}
\lambda \int_{B_{s}\left(x_{0}\right)}\left|D^{m} \varphi\right|^{2} \mathrm{~d} \mathcal{L}^{n} \leqslant & \frac{L}{2} \int_{B_{s}\left(x_{0}\right) \backslash B_{t}\left(x_{0}\right)}\left|D^{m} v\right|^{2} \mathrm{~d} \mathcal{L}^{n} \\
& +\frac{3}{2} L\left|\int_{B_{s}\left(x_{0}\right) \backslash B_{t}\left(x_{0}\right)} \sum_{k=0}^{m}\left(\begin{array}{c}
m \\
k
\end{array}\right) D^{m-k}(1-\eta) \otimes D^{k} v \mathrm{~d} \mathcal{L}^{n}\right|^{2} \\
\leqslant & \frac{3 m+4}{2} L \int_{B_{s}\left(x_{0}\right) \backslash B_{t}\left(x_{0}\right)}\left|D^{m} v\right|^{2} \mathrm{~d} \mathcal{L}^{n} \\
& +\frac{3}{2} L(m+1) \sum_{k=0}^{m-1}\left(\begin{array}{c}
m \\
k
\end{array}\right)^{2} \int_{B_{s}\left(x_{0}\right) \backslash B_{t}\left(x_{0}\right)}\left|D^{m-k}(1-\eta)\right|^{2}\left|D^{k} v\right|^{2} \mathrm{~d} \mathcal{L}^{n} \\
\leqslant & \frac{3 m+4}{2} \int_{B_{s}\left(x_{0}\right) \backslash B_{t}\left(x_{0}\right)}\left|D^{m} v\right|^{2} \mathrm{~d} \mathcal{L}^{n} \\
& +\frac{3}{2} L(m+1) \sum_{k=0}^{m-1}(m)^{2}\left(C_{\eta}(s-t)\right]^{2(k-m)} \int_{B_{s}\left(x_{0}\right) \backslash B_{t}\left(x_{0}\right)}\left|D^{k} v\right|^{2} \mathrm{~d} \mathcal{L}^{n} .
\end{aligned}
$$

Using $\int_{B_{t}\left(x_{0}\right)}\left|D^{m} v\right|^{2} \mathrm{~d} \mathcal{L}^{n} \leqslant \int_{B_{s}\left(x_{0}\right)}\left|D^{m} \varphi\right|^{2} \mathrm{~d} \mathcal{L}^{n}$ and "filling the hole" on the right side we conclude:

$$
\begin{aligned}
& \int_{B_{t}\left(x_{0}\right)}\left|D^{m} v\right|^{2} \leqslant \frac{(3 m+4) L}{(3 m+4) L+2 \lambda} \int_{B_{s}\left(x_{0}\right)}\left|D^{m} v\right|^{2} \mathrm{~d} \mathcal{L}^{n} \\
& +\frac{3 L(m+1)}{(3 m+4) L+2 \lambda} \sum_{k=0}^{m-1}\left(\begin{array}{c}
m \\
k
\end{array}\right)^{2}\left[C_{\eta}(s-t)\right]^{2(k-m)} \int_{B_{s}\left(x_{0}\right) \backslash B_{t}\left(x_{0}\right)}\left|D^{k} v\right|^{2} \mathrm{~d} \mathcal{L}^{n} .
\end{aligned}
$$

Now we use the following technical lemma which is an extension of Lemma 5.1 in [12]:

LEMMA 4. - Let $0<\theta<1, A_{k} \geqslant 0$ and $\alpha_{k}>0$ for $k=0,1, \ldots, l$ and $f \geqslant 0 a$ bounded function satisfying

$$
f(t) \leqslant \theta f(s)+\sum_{k=0}^{l} A_{k}(s-t)^{-\alpha_{k}}
$$

for all $0<r \leqslant t<s \leqslant \rho$. Then there exists a constant $C_{\text {tech }}=C_{\text {tech }}\left(\alpha_{0}, \ldots, \alpha_{l}, \theta\right)$ such that

$$
f(r) \leqslant C_{\text {tech }}\left(\sum_{k=0}^{l} A_{k}(\rho-r)^{-\alpha_{k}}\right) .
$$

Apply this technical lemma to (14) with $l=m-1$ and then choose $r=\rho / 2$. This guarantees the existence of a constant $\widetilde{C}_{c a c}=\widetilde{C}_{c a c}(m, L, \lambda)$ such that the asserted Cac-

\footnotetext{
${ }^{2}$ This easily follows from the product formula $D(f \otimes g)=D f \otimes g+f \otimes D g$ for tensor products.
} 
cioppoli inequality holds. The "moreover"-case is a direct implication from Poincarés inequality Lemma 1.

Proof of Lemma 4. - For $0<\tau<1$ satisfying $\tau^{-\alpha_{k}} \theta<1$ for $k=0, \ldots, l$ (i.e. $1>$ $\left.\tau^{\max \left(\alpha_{k}\right)}>\theta\right)$ define the recursive sequence $t_{0}=r$ and $t_{n+1}=t_{n}+(1-\tau) \tau^{n}(\rho-r) \leqslant \rho$. We get

$$
\begin{aligned}
f(r) & =f\left(t_{0}\right) \leqslant \theta^{n} f\left(t_{n}\right)+\sum_{j=0}^{n-1} \theta^{j}\left(\sum_{k=0}^{l} A_{k}\left[(1-\tau) \tau^{j}(\rho-r)\right]^{-\alpha_{k}}\right) \\
& =\theta^{n} f\left(t_{n}\right)+\sum_{k=0}^{l} A_{k}[(1-\tau)(\rho-r)]^{-\alpha_{k}} \sum_{j=0}^{n-1}\left(\theta \tau^{-\alpha_{k}}\right)^{j} .
\end{aligned}
$$

Define $C_{\text {tech }}=\max _{k=0, \ldots, l} \frac{(1-\tau)^{-\alpha_{k}}}{1-\theta \tau^{-\alpha_{k}}}$ and get the desired estimate letting $n \rightarrow \infty$.

Now we state a Caccioppoli inequality in the case $p \geqslant 2$.

LEMMA 5. - Let $u \in W^{m, p}\left(\Omega, \mathbb{R}^{N}\right)$ be a minimizer of the functional $\int_{\Omega} f\left(D^{m} u\right) \mathrm{d} \mathcal{L}^{n}$, where $f$ satisfies $(\mathrm{H} 3)$ and $(\mathrm{H} 4)$. Then for each $M>0$ there exists a constant $\widetilde{C}_{c a c}=$ $\widetilde{C}_{c a c}(L, \lambda, p, m, M)$ such that

$$
\begin{aligned}
& \int_{B_{\rho / 2}\left(x_{0}\right)}\left[\left|D^{m}(u-P)\right|^{2}+\left|D^{m}(u-P)\right|^{p}\right] \mathrm{d} \mathcal{L}^{n} \\
& \leqslant \widetilde{C}_{c a c}^{2} \sum_{k=0}^{m-1}\left[\rho^{2(k-m)} \int_{B_{\rho}\left(x_{0}\right)}\left|D^{k}(u-P)\right|^{2} \mathrm{~d} \mathcal{L}^{n}+\rho^{p(k-m)} \int_{B_{\rho}\left(x_{0}\right)}\left|D^{k}(u-P)\right|^{p} \mathrm{~d} \mathcal{L}^{n}\right]
\end{aligned}
$$

for all balls $B_{\rho}\left(x_{0}\right) \Subset \Omega$ and all polynomials $P: \mathbb{R}^{N} \supset B_{\rho}\left(x_{0}\right) \rightarrow \mathbb{R}^{N}$ of degree $\leqslant m$ satisfying

$$
\left|D^{m} P\right| \leqslant M \text {. }
$$

Moreover, if $\int_{B_{\rho}\left(x_{0}\right)} D^{k}(u-P) \mathrm{d} \mathcal{L}^{n}=0$ for $0 \leqslant k \leqslant m-2$, then

$$
\begin{aligned}
& \int_{B_{\rho / 2}\left(x_{0}\right)}\left[\left|D^{m}(u-P)\right|^{2}+\left|D^{m}(u-P)\right|^{p}\right] \mathrm{d} \mathcal{L}^{n} \\
& \leqslant C_{c a c}^{2}\left[\rho^{-2} \int_{B_{\rho}\left(x_{0}\right)}\left|D^{m-1}(u-P)\right|^{2} \mathrm{~d} \mathcal{L}^{n}+\rho^{-p} \int_{B_{\rho}\left(x_{0}\right)}\left|D^{m-1}(u-P)\right|^{p} \mathrm{~d} \mathcal{L}^{n}\right],
\end{aligned}
$$

where $C_{c a c}=C_{c a c}(L, \lambda, p, m, n, N, M)$.

Proof. - Take $\varphi, \psi, v$ and $A$ as in the proof of Lemma 3. Using the quasiconvexity condition (H3), (13) and the minimality of $u$ we obtain:

$$
\begin{aligned}
\lambda \int_{B_{s}\left(x_{0}\right)}\left[\left|D^{m} \varphi\right|^{2}+\left|D^{m} \varphi\right|^{p}\right] \mathrm{d} \mathcal{L}^{n} & \leqslant \int_{B_{s}\left(x_{0}\right)}\left[f\left(A+D^{m} \varphi\right)-f(A)\right] \mathrm{d} \mathcal{L}^{n} \\
& =\int_{B_{s}\left(x_{0}\right)}\left[f\left(D^{m} u-D^{m} \psi\right)-f(A)\right] \mathrm{d} \mathcal{L}^{n}
\end{aligned}
$$




$$
\begin{aligned}
& \leqslant \int_{B_{s}\left(x_{0}\right)}\left[f\left(D^{m} u-D^{m} \psi\right)-f\left(D^{m} u\right)\right] \mathrm{d} \mathcal{L}^{n} \\
& +\int_{B_{s}\left(x_{0}\right)}\left[f\left(D^{m} u\right)-f\left(D^{m} u-D^{m} \varphi\right)\right] \mathrm{d} \mathcal{L}^{n} \\
& +\int_{B_{s}\left(x_{0}\right)}\left[f\left(D^{m} u-D^{m} \varphi\right)-f(A)\right] \mathrm{d} \mathcal{L}^{n} \\
& \leqslant \int_{B_{s}\left(x_{0}\right)}\left[f\left(D^{m} u-D^{m} \psi\right)-f\left(D^{m} u\right)\right] \mathrm{d} \mathcal{L}^{n} \\
& +\int_{B_{s}\left(x_{0}\right)}\left[f\left(A+D^{m} \psi\right)-f(A)\right] \mathrm{d} \mathcal{L}^{n} .
\end{aligned}
$$

Using Taylor's formular, we can further derive

$$
\begin{aligned}
& \lambda \int_{B_{s}\left(x_{0}\right)}\left[\left|D^{m} \varphi\right|^{2}+\left|D^{m} \varphi\right|^{p}\right] \mathrm{d} \mathcal{L}^{n} \\
& \leqslant \int_{B_{s}\left(x_{0}\right)}\left[-D f\left(D^{m} u\right) D^{m} \psi+\int_{0}^{1}(1-t) D^{2} f\left(t\left(D^{m} u-D^{m} \psi\right)\right.\right. \\
& \left.\left.+(1-t) D^{m} u\right)\left(D^{m} \psi, D^{m} \psi\right) \mathrm{d} t\right] \mathrm{d} \mathcal{L}^{n} \\
& +\int_{B_{s}\left(x_{0}\right)}\left[D f(A) D^{m} \psi+\int_{0}^{1}(1-t) D^{2} f\left(t\left(D^{m} \psi+A\right)+(1-t) A\right)\left(D^{m} \psi, D^{m} \psi\right) \mathrm{d} t\right] \mathrm{d} \mathcal{L}^{n} .
\end{aligned}
$$

From the estimate

$$
(a+b)^{p-2} \leqslant \begin{cases}2^{p-2}\left(a^{p-2}+b^{p-2}\right), & 0<p-2 \leqslant 1 \\ 2^{p-3}\left(a^{p-2}+b^{p-2}\right), & p-2 \geqslant 1\end{cases}
$$

for $a, b \geqslant 0$, hypothesis $(\mathrm{H} 4), \Psi_{\mid B_{t}\left(x_{0}\right)}=0, v=u-P$ and $|A| \leqslant M$ we now get

$$
\begin{aligned}
& \lambda \int_{B_{s}\left(x_{0}\right)}\left[\left|D^{m} \varphi\right|^{2}+\left|D^{m} \varphi\right|^{p}\right] \mathrm{d} \mathcal{L}^{n} \\
& \leqslant L \int_{B_{s}\left(x_{0}\right)} \int_{0}^{1}\left(1+\left|t A+(1-t) D^{m} u\right|^{p-2}\right)\left|D^{m} u-A\right|\left|D^{m} \psi\right| \mathrm{d} t \mathrm{~d} \mathcal{L}^{n} \\
& \quad+L \int_{B_{s}\left(x_{0}\right)} \int_{0}^{1}\left(1+\left|D^{m} u-t D^{m} \psi\right|^{p-2}\right)\left|D^{m} \psi\right|^{2} \mathrm{~d} t \mathrm{~d} \mathcal{L}^{n}
\end{aligned}
$$




$$
\begin{aligned}
& +L \int_{B_{s}\left(x_{0}\right)} \int_{0}^{1}\left(1+\left|A+t D^{m} \psi\right|^{p-2}\right)\left|D^{m} \psi\right|^{2} \mathrm{~d} t \mathrm{~d} \mathcal{L}^{n} \\
\leqslant & 2^{p-2} L \int_{B_{s}\left(x_{0}\right)}\left[\left(1+M^{p-2}+\left|D^{m} u\right|^{p-2}\right)\left|D^{m} u-A\right|\left|D^{m} \psi\right|\right. \\
& \left.+\left(1+M^{p-2}+\left|D^{m} u\right|^{p-2}+\left|D^{m} \psi\right|^{p-2}\right)\left|D^{m} \psi\right|^{2}\right] \mathrm{d} \mathcal{L}^{n} \\
\leqslant & 4^{p-2} L \int_{B_{s}\left(x_{0}\right) \backslash B_{t}\left(x_{0}\right)}\left[\left(1+M^{p-2}+\left|D^{m} v\right|^{p-2}\right)\left|D^{m} v\right|\left|D^{m} \psi\right|\right. \\
& \left.+\left(1+M^{p-2}+\left|D^{m} v\right|^{p-2}+\left|D^{m} \psi\right|^{p-2}\right)\left|D^{m} \psi\right|^{2}\right] \mathrm{d} \mathcal{L}^{n} .
\end{aligned}
$$

Using Hölder's and Young's inequality we obtain

$$
\begin{aligned}
& \lambda \int_{B_{s}\left(x_{0}\right)}\left[\left|D^{m} \varphi\right|^{2}+\left|D^{m} \varphi\right|^{p}\right] \mathrm{d} \mathcal{L}^{n} \\
& \leqslant \frac{3}{2} 4^{p-2} L\left(1+M^{p-2}\right)\left[\int_{B_{s}\left(x_{0}\right) \backslash B_{t}\left(x_{0}\right)}\left(\left|D^{m} v\right|^{2}+\left|D^{m} v\right|^{p}\right) \mathrm{d} \mathcal{L}^{n}\right. \\
& \left.\quad+\int_{B_{s}\left(x_{0}\right) \backslash B_{t}\left(x_{0}\right)}\left(\left|D^{m} \psi\right|^{2}+\left|D^{m} \psi\right|^{p}\right) \mathrm{d} \mathcal{L}^{n}+\int_{B_{s}\left(x_{0}\right) \backslash B_{t}\left(x_{0}\right)}\left(\left|D^{m} v\right|^{p}+\left|D^{m} \psi\right|^{p}\right) \mathrm{d} \mathcal{L}^{n}\right] \\
& \leqslant 3 \cdot 4^{p-2} L\left(1+M^{p-2}\right)\left[\int_{B_{s}\left(x_{0}\right) \backslash B_{t}\left(x_{0}\right)}\left(\left|D^{m} v\right|^{2}+\left|D^{m} v\right|^{p}\right) \mathrm{d} \mathcal{L}^{n}\right. \\
& \left.+\int_{B_{s}\left(x_{0}\right) \backslash B_{t}\left(x_{0}\right)}\left(\left|D^{m} \psi\right|^{2}+\left|D^{m} \psi\right|^{p}\right) \mathrm{d} \mathcal{L}^{n}\right] .
\end{aligned}
$$

Since $D^{m} v=D^{m} \varphi$ on $B_{t}\left(x_{0}\right)$ we find

$$
\begin{aligned}
\lambda \int_{B_{t}\left(x_{0}\right)} & {\left[\left|D^{m} v\right|^{2}+\left|D^{m} v\right|^{p}\right] \mathrm{d} \mathcal{L}^{n} } \\
\leqslant & 3 \cdot 4^{p-2} L(m+1)^{p-1}\left(1+M^{p-2}\right) \cdot\left[\int_{B_{s}\left(x_{0}\right) \backslash B_{t}\left(x_{0}\right)}\left(\left|D^{m} v\right|^{2}+\left|D^{m} v\right|^{p}\right) \mathrm{d} \mathcal{L}^{n}\right. \\
& +\sum_{k=0}^{m-1}\left(\begin{array}{c}
m \\
k
\end{array}\right)^{2} C_{\eta}^{2}(s-t)^{2(k-m)} \int_{B_{s}\left(x_{0}\right)}\left|D^{k} v\right|^{2} \mathrm{~d} \mathcal{L}^{n} \\
& \left.+\sum_{k=0}^{m-1}\left(\begin{array}{c}
m \\
k
\end{array}\right)^{p} C_{\eta}^{p}(s-t)^{p(k-m)} \int_{B_{s}\left(x_{0}\right)}\left|D^{k} v\right|^{p} \mathrm{~d} \mathcal{L}^{n}\right] .
\end{aligned}
$$

"Filling the hole" on the right-hand side leads to 


$$
\begin{aligned}
& \int_{B_{t}\left(x_{0}\right)}\left[\left|D^{m} v\right|^{2}+\left|D^{m} v\right|^{p}\right] \mathrm{d} \mathcal{L}^{n} \\
& \leqslant \frac{3 \cdot 4^{p-2} L(m+1)^{p-1}\left(1+M^{p-2}\right)}{\lambda+3 \cdot 4^{p-2} L(m+1)^{p-1}\left(1+M^{p-2}\right)} \int_{B_{s}\left(x_{0}\right)}\left(\left|D^{m} v\right|^{2}+\left|D^{m} v\right|^{p}\right) \mathrm{d} \mathcal{L}^{n} \\
& \quad+C_{\eta}^{p}(m !)^{p} \sum_{k=0}^{m-1}\left[(s-t)^{2(k-m)} \int_{B_{s}\left(x_{0}\right)}\left|D^{k} v\right|^{2} \mathrm{~d} \mathcal{L}^{n}+(s-t)^{p(k-m)} \int_{B_{s}\left(x_{0}\right)}\left|D^{k} v\right|^{p} \mathrm{~d} \mathcal{L}^{n}\right] .
\end{aligned}
$$

Here we have used $C_{\eta}^{2} \leqslant C_{\eta}^{p}$ and

$$
\frac{C_{\eta}^{p}(m !)^{p} \cdot 3 \cdot 4^{p-2} L(m+1)^{p-1}\left(1+M^{p-2}\right)}{\lambda+3 \cdot 4^{p-2} L(m+1)^{p-1}\left(1+M^{p-2}\right)} \leqslant C_{\eta}^{p}(m !)^{p} .
$$

Now, an application of Lemma 4 with

$$
\theta=\frac{3 \cdot 4^{p-2} L(m+1)^{p-1}\left(1+M^{p-2}\right)}{\lambda+3 \cdot 4^{p-2} L(m+1)^{p-1}\left(1+M^{p-2}\right)}
$$

yields the desired estimate

$$
\begin{aligned}
& \int_{B_{\rho / 2}}\left[\left|D^{m}(u-P)\right|^{2}+\left|D^{m}(u-P)\right|^{p}\right] \mathrm{d} \mathcal{L}^{n} \\
& \leqslant C_{c a c}^{2}(L, \lambda, p, m, M) \sum_{k=0}^{m-1}\left[\rho^{2(k-m)} \int_{B_{\rho}}\left|D^{k}(u-P)\right|^{2} \mathrm{~d} \mathcal{L}^{n}\right. \\
& \left.\quad+\rho^{p(k-m)} \int_{B_{\rho}}\left|D^{k}(u-P)\right|^{p} \mathrm{~d} \mathcal{L}^{n}\right] .
\end{aligned}
$$

The dependence of $C_{c a c}$ on $M$ follows from the dependence of $C_{t e c h}$ on $\theta$. Note, that $M \rightarrow \infty$ implies $\theta \rightarrow 1$ and $C_{\text {tech }} \rightarrow \infty$. The second estimate follows with an application of Poincaré's inequality (cf. Lemma 1).

\section{The $(\mathcal{A}, m)$-harmonic approximation lemma}

The result of this section, the $(\mathcal{A}, m)$-harmonic approximation lemma, is central to our technique. In the case $m=1$ the result was given in [8, Lemma 3.3] (confer [21], Section 1.6 for the case of Laplace's equation and harmonic approximation).

Lemma $6((\mathcal{A}, m)$-harmonic approximation lemma). - For any given $\varepsilon>0$ there exists $\delta=\delta(n, N, \lambda, L, m, \varepsilon) \in] 0,1]$ with the following property: for any given $\mathcal{A} \in$ $\bigodot^{2}\left(\bigodot^{m}\left(\mathbb{R}^{n}, \mathbb{R}^{N}\right), \mathbb{R}\right)$ satisfying

$$
\int_{\Omega} \mathcal{A}\left(D^{m} w, D^{m} w\right) \mathrm{d} \mathcal{L}^{n} \geqslant \lambda \int_{\Omega}\left|D^{m} w\right|^{2} \mathrm{~d} \mathcal{L}^{n}
$$


for all $w \in W_{0}^{m, 2}\left(\Omega, \mathbb{R}^{N}\right)$ and

$$
\mathcal{A}(A, B) \leqslant L|A||B|
$$

for all $A, B \in \bigodot^{m}\left(\mathbb{R}^{n}, \mathbb{R}^{N}\right)$, and for any $g \in W^{m, 2}\left(B_{\rho}\left(x_{0}\right), \mathbb{R}^{N}\right)$ satisfying

$$
\begin{gathered}
f_{B_{\rho}\left(x_{0}\right)}\left|D^{m} g\right|^{2} \leqslant 1 \quad \text { and } \\
\left|f_{B_{\rho}\left(x_{0}\right)} \mathcal{A}\left(D^{m} g, D^{m} \varphi\right) \mathrm{d} \mathcal{L}^{n}\right| \leqslant \delta \sup _{B_{\rho}\left(x_{0}\right)}\left|D^{m} \varphi\right| \quad \text { for all } \varphi \in C_{0}^{\infty}\left(B_{\rho}\left(x_{0}\right), \mathbb{R}^{N}\right),
\end{gathered}
$$

there exists a function $v \in W^{m, 2}\left(B_{\rho}\left(x_{0}\right), \mathbb{R}^{N}\right)$ with the following properties:

$$
\begin{gathered}
f_{B_{\rho}\left(x_{0}\right)}\left|D^{m} v\right|^{2} \mathrm{~d} \mathcal{L}^{n} \leqslant 1, \\
\int_{B_{\rho}\left(x_{0}\right)} \mathcal{A}\left(D^{m} v, D^{m} \varphi\right) \mathrm{d} \mathcal{L}^{n}=0
\end{gathered}
$$

for all $\varphi \in C_{0}^{m}\left(B_{\rho}\left(x_{0}\right), \mathbb{R}^{N}\right)$ and

$$
\rho^{-2} f_{B_{\rho}\left(x_{0}\right)} \sum_{\gamma=0}^{m-1}\left|D^{\gamma}(v-g)\right|^{2} \mathrm{~d} \mathcal{L}^{n} \leqslant \varepsilon .
$$

Proof. - We assume first that $x_{0}=0$ and $\rho=1, B_{1}(0)=B$. If the conclusions of the theorem were false, we could find $\varepsilon>0$ such that for every $k \in \mathbb{N}$ there exist bilinear forms $\mathcal{A}_{k}: \bigodot^{m}\left(\mathbb{R}^{n}, \mathbb{R}^{N}\right) \rightarrow \mathbb{R}$ and functions $g_{k} \in W^{m, 2}\left(B, \mathbb{R}^{N}\right)$, so that for all $k \in \mathbb{N}$ we would have

$$
\begin{gathered}
f_{B}\left|D^{m} g_{k}\right|^{2} \mathrm{~d} \mathcal{L}^{n} \leqslant 1, \\
f_{B} \mathcal{A}_{k}\left(D^{m} g_{k}, D^{m} \varphi\right) \leqslant \frac{1}{k} \sup _{B}\left|D^{m} \varphi\right| \quad \text { for all } \varphi \in C_{0}^{\infty}\left(B, \mathbb{R}^{N}\right), \\
\text { but } \quad \int_{B}^{m-1}\left|D_{\gamma=0}^{\gamma}\left(v-g_{k}\right)\right|^{2} \mathrm{~d} \mathcal{L}^{n} \geqslant \varepsilon
\end{gathered}
$$

for all $v \in \mathcal{H}_{k}$. Here $\mathcal{H}_{k}$ denotes the nonempty set of all $h \in W^{m, 2}\left(B, \mathbb{R}^{N}\right)$ for which

$$
\begin{gathered}
f_{B}\left|D^{m} h\right|^{2} \mathrm{~d} \mathcal{L}^{n} \leqslant 1 \quad \text { and } \\
f_{B} \mathcal{A}_{k}\left(D^{m} h, D^{m} \varphi\right)=0 \text { for all } \varphi \in C_{0}^{\infty}\left(B, \mathbb{R}^{N}\right) ;
\end{gathered}
$$

the second of these conditions expresses the fact that $h$ is $\left(\mathcal{A}_{k}, m\right)$-harmonic on $B$. 
Without loss of generality we can assume

$$
f_{B} D^{\gamma} g_{k} \mathrm{~d} \mathcal{L}^{n}=0
$$

for all $0 \leq \gamma \leqslant m-1$ and all $k \in \mathbb{N}$ (by simply replacing $g_{k}$ with $g_{k}-P_{k}$ where $P_{k}$ is the unique polynomial of degree at most $m-1$ satiyfying $\int_{B} D^{\gamma}\left(g_{k}-P_{k}\right) \mathrm{d} \mathcal{L}^{n}=0$ for $0 \leqslant \gamma \leqslant m-1$; see Lemma 1.1 in [13]). From (18) and (21) we have, using Poincaré's inequality, that $\left\{\left\|g_{k}\right\|_{W^{m, 2}\left(B, \mathbb{R}^{N}\right)}\right\}$ is uniformly bounded for all $k \in \mathbb{N}$. From Rellich's lemma and the upper bound (17) for $\mathcal{A}_{k}$ we have the existence of a function $g \in W^{m, 2}\left(B, \mathbb{R}^{N}\right)$ and a bilinear function $\mathcal{A} \in \bigodot^{2}\left(\bigodot^{m}\left(\mathbb{R}^{n}, \mathbb{R}^{N}\right), \mathbb{R}\right)$ such that, after passage to subsequence (which we label again with $k$ ), there holds:

$$
\begin{aligned}
& g_{k} \rightarrow g \quad \text { weakly in } W^{m, 2}\left(B, \mathbb{R}^{N}\right), \\
& g_{k} \rightarrow g \quad \text { in } W^{m-1,2}\left(B, \mathbb{R}^{N}\right) \quad \text { and } \\
& \mathcal{A}_{k} \rightarrow \mathcal{A} \quad \text { in } \bigodot^{2}\left(\bigodot^{m}\left(\mathbb{R}^{n}, \mathbb{R}^{N}\right), \mathbb{R}\right) .
\end{aligned}
$$

Hence we see from (18)

$$
f_{B}\left|D^{m} g\right|^{2} \mathrm{~d} \mathcal{L}^{n} \leqslant 1 .
$$

Further we have for every $k \in \mathbb{N}$ and every $\varphi \in C_{0}^{\infty}\left(B, \mathbb{R}^{N}\right)$

$$
\begin{aligned}
f_{B} \mathcal{A}\left(D^{m} g, D^{m} \varphi\right) \mathrm{d} \mathcal{L}^{n} \leqslant & f_{B} \mathcal{A}\left(D^{m} g-D^{m} g_{k}, D^{m} \varphi\right) \mathrm{d} \mathcal{L}^{n} \\
& +f_{B}\left(\mathcal{A}-\mathcal{A}_{k}\right)\left(D^{m} g_{k}, D^{m} \varphi\right) \mathrm{d} \mathcal{L}^{n}+f_{B} \mathcal{A}_{k}\left(D^{m} g_{k}, D^{m} \varphi\right) \mathrm{d} \mathcal{L}^{n},
\end{aligned}
$$

where the terms on the right side vanish via (22), (24), (25) and (19). Therefore $g$ is $(\mathcal{A}, m)$-harmonic in $B$. We denote by $V_{k} \in W^{m, 2}\left(B, \mathbb{R}^{N}\right)$ the unique solution of the Dirichlet problem

$$
\begin{gathered}
f \mathcal{A}_{k}\left(D^{m} V_{k}, D^{m} \varphi\right) \mathrm{d} \mathcal{L}^{n}=0 \quad \text { for all } \varphi \in C_{0}^{\infty}\left(B, \mathbb{R}^{N}\right), \\
D^{\gamma}\left(V_{k}-g\right) \in W_{0}^{m, 2}\left(B, \mathbb{R}^{N}\right) \quad \text { for } 0 \leqslant \gamma \leqslant m-1 .
\end{gathered}
$$

In view of (16), (24) and the $(\mathcal{A}, m)$-harmonicity of $g$ we then have:

$$
\begin{aligned}
\lambda \int_{B}\left|D^{m}\left(V_{k}-g\right)\right|^{2} \mathrm{~d} \mathcal{L}^{n} & \leqslant \int_{B} \mathcal{A}_{k}\left(D^{m}\left(V_{k}-g\right), D^{m}\left(V_{k}-g\right)\right) \mathrm{d} \mathcal{L}^{n} \\
& =-\int_{B} \mathcal{A}_{k}\left(D^{m} g, D^{m}\left(V_{k}-g\right)\right) \mathrm{d} \mathcal{L}^{n} \\
& =\int_{B}\left(\mathcal{A}-\mathcal{A}_{k}\right)\left(D^{m} g, D^{m}\left(V_{k}-g\right)\right) \mathrm{d} \mathcal{L}^{n}
\end{aligned}
$$




$$
\begin{aligned}
& \leqslant\left|A-A_{k}\right| \int_{B}\left|D^{m} g\right|\left|D^{m}\left(V_{k}-g\right)\right| \mathrm{d} \mathcal{L}^{n} \\
& \leqslant\left|A-A_{k}\right|\left(\int_{B}\left|D^{m} g\right|^{2} \mathrm{~d} \mathcal{L}^{n}\right)^{1 / 2}\left(\int_{B}\left|D^{m}\left(V_{k}-g\right)\right|^{2} \mathrm{~d} \mathcal{L}^{n}\right)^{1 / 2} .
\end{aligned}
$$

In view of (24) and (25) this implies strong convergence

$$
V_{k} \rightarrow g \quad \text { in } W^{2, m}\left(B, \mathbb{R}^{N}\right)
$$

We now define $v_{k}:=V_{k} / b_{k}$ where $b_{k}=\max \left\{1,\left\|D^{m} V_{k}\right\|_{L^{2}\left(B, \mathbb{R}^{N}\right)}\right\}$; then $v_{k} \in \mathcal{H}_{k}$ with

$$
\left\|v_{k}-g_{k}\right\|_{W^{m-1,2}} \leqslant\left\|v_{k}-V_{k}\right\|_{W^{m-1,2}}+\left\|V_{k}-g\right\|_{W^{m-1,2}}+\left\|g-g_{k}\right\|_{W^{m-1,2}} \rightarrow 0 .
$$

From (27) and (22) we see that the second and the third term on the right-hand side approach 0 as $k \rightarrow \infty$. From (27) we see $b_{k} \rightarrow 1$ as $k \rightarrow \infty$. Together with (24) this implies that the first term of the right-hand side also tends to 0 as $k \rightarrow \infty$. But this contradicts (20).

The general result follows from a simple scaling arguments.

The next result is a standard estimate for solutions of systems with constant coefficients (see [5,6]).

LEMma 7. - Consider $\mathcal{A} \in \bigodot^{2}\left(\bigodot^{m}\left(\mathbb{R}^{n}, R^{N}\right), \mathbb{R}\right)$ satifying (16) and (17) and $h \in$ $W^{m, 2}\left(B_{\rho}\left(x_{0}\right), \mathbb{R}^{N}\right)$ with $\int_{B_{\rho}\left(x_{0}\right)} \mathcal{A}\left(D^{m} h, D^{m} \varphi\right) \mathrm{d} \mathcal{L}^{n}=0$ for all $\varphi \in C_{0}^{\infty}\left(B_{\rho}\left(x_{0}\right), \mathbb{R}^{N}\right)$. Then there exists a constant $C_{\text {harm }}=C_{\text {harm }}(n, N, m, \lambda, \Lambda)$ such that the following estimate holds:

$$
\rho^{-2} \sup _{B_{\rho / 2}\left(x_{0}\right)}\left|D^{m} h\right|^{2}+\sup _{B_{\rho / 2}\left(x_{0}\right)}\left|D^{m+1} h\right|^{2} \leqslant C_{h a r m}^{2} \rho^{-2} f_{B_{\rho}\left(x_{0}\right)}\left|D^{m} h\right|^{2} \mathrm{~d} \mathcal{L}^{n} .
$$

The last lemma in this section is required in order to be able to apply the $(\mathcal{A}, m)$ harmonic approximation technique. For a ball $B_{\rho}\left(x_{0}\right) \subset \Omega, u \in W^{m, 2}\left(B_{\rho}\left(x_{0}\right), \mathbb{R}^{N}\right)$ resp. $u \in W^{m, p}\left(B_{\rho}\left(x_{0}\right), \mathbb{R}^{N}\right)$ and a polynomial $P: B_{\rho}\left(x_{0}\right) \rightarrow \mathbb{R}^{N}$ of degree $\leqslant m$ we define

$$
\begin{gathered}
\Phi_{2}\left(x_{0}, \rho, P\right):=\left(\underset{B_{\rho}\left(x_{0}\right)}{f}\left|D^{m}(u-P)\right|^{2} \mathrm{~d} \mathcal{L}^{n}\right)^{1 / 2}, \\
\Phi_{p}\left(x_{0}, \rho, P\right):=\left(f_{B_{\rho}\left(x_{0}\right)}\left|D^{m}(u-P)\right|^{2} \mathrm{~d} \mathcal{L}^{n}+f_{B_{\rho}\left(x_{0}\right)}\left|D^{m}(u-P)\right|^{p} \mathrm{~d} \mathcal{L}^{n}\right)^{1 / 2} .
\end{gathered}
$$

LEMMA 8. -

(i) Let $u \in W^{m, 2}\left(\Omega, \mathbb{R}^{N}\right)$ be a weak solution of the Euler equations (10) and assume that $f$ satisfies hypothesis (H2). Then for every polynomial $P$ of degree at most $m$ and every ball $B_{\rho}\left(x_{0}\right) \Subset \Omega$ there holds: 


$$
\begin{aligned}
& \left|\int_{B_{\rho}\left(x_{0}\right)} D^{2} f\left(D^{m} P\right)\left(D^{m}(u-P), D^{m} \varphi\right) \mathrm{d} \mathcal{L}^{n}\right| \\
& \quad \leqslant C_{e u} \sqrt{v\left(\Phi_{2}\left(x_{0}, \rho, P\right)\right)} \Phi_{2}\left(x_{0}, \rho, P\right) \sup _{B_{\rho}\left(x_{0}\right)}\left|D^{m} \varphi\right|
\end{aligned}
$$

for all $\varphi \in C_{0}^{\infty}\left(B_{\rho}\left(x_{0}\right), \mathbb{R}^{N}\right)$ with a constant $C_{e u}=\sqrt{2 L}=C_{e u}(L)$. (The constant $L$ is from hypothesis $(\mathrm{H} 2)$ and $v$ is the modulus of continuity for $D^{2} f$, see Remark 4.)

(ii) Let $u \in W^{m, p}\left(\Omega, \mathbb{R}^{N}\right)$ be a weak solution of the Euler equations (10) and let $f$ satisfy hypotheses (H4). Then for every $M>0$ there exists $C_{e u}=C_{e u}(p, L, M)$ (we can choose $C_{e u}=2^{p+1} L^{\frac{p-1}{p}}\left(1+M^{p-2}\right)$ ) such that

$$
\begin{aligned}
& \left|\int_{B_{\rho}\left(x_{0}\right)} D^{2} f\left(D^{m} P\right)\left(D^{m}(u-P), D^{m} \varphi\right) \mathrm{d} \mathcal{L}^{n}\right| \\
& \quad \leqslant C_{e u} v_{M}\left(\Phi_{p}\left(x_{0}, \rho, P\right)\right)^{1 / p} \Phi_{p}\left(x_{0}, \rho, P\right) \sup _{B_{\rho}\left(x_{0}\right)}\left|D^{m} \varphi\right|
\end{aligned}
$$

for all $B_{\rho}\left(x_{0}\right) \Subset \Omega, \varphi \in C_{0}^{\infty}\left(B_{\rho}\left(x_{0}\right), \mathbb{R}^{N}\right)$ and every polynomial $P$ of degree at most $m$ with

$$
\Phi_{p}\left(x_{0}, \rho, P\right) \leqslant 1 \text { and }\left|D^{m} P\right| \leqslant M .
$$

(The constant L is from hypothesis $(\mathrm{H} 4)$ and $v_{M}$ is from Remark 4.)

Proof. - From the Euler equations (10) and the fact that $D^{m} P$ is constant, we get

$$
\begin{aligned}
0 & =f_{B_{\rho}\left(x_{0}\right)}\left(D f\left(D^{m} u\right)-D f\left(D^{m} P\right)\right) D^{m} \varphi \mathrm{d} \mathcal{L}^{n} \\
& =f_{B_{\rho}\left(x_{0}\right)} \int_{0}^{1} \frac{d}{d t} D f\left(D^{m} P-t D^{m}(u-P)\right) \mathrm{d} t D^{m} \varphi \mathrm{d} \mathcal{L}^{n} \\
& =f_{B_{\rho}\left(x_{0}\right)} \int_{0}^{1} D^{2} f\left(D^{m} P-t D^{m}(u-P)\right) \mathrm{d} t\left(D^{m}(u-P), D^{m} \varphi\right) \mathrm{d} \mathcal{L}^{n} .
\end{aligned}
$$

In view of hypothesis (H2) this leads to, using the statement in Remark 4, Hölder's and Jensen's inequality,

$$
\begin{aligned}
& \left|\underset{B_{\rho}\left(x_{0}\right)}{f} D^{2} f\left(D^{m} P\right)\left(D^{m}(u-P), D^{m} \varphi\right) \mathrm{d} \mathcal{L}^{n}\right| \\
& =\mid f_{B_{\rho}\left(x_{0}\right)} \int_{0}^{1}\left(D^{2} f\left(D^{m} P\right)-D^{2} f\left(D^{m} P-t D^{m}(u-P)\right)\right) \mathrm{d} t \\
& \quad \times\left(D^{m}(u-P), D^{m} \varphi\right) \mathrm{d} \mathcal{L}^{n} \mid
\end{aligned}
$$




$$
\begin{aligned}
\leqslant & \sqrt{2 L} f \int_{B_{\rho}\left(x_{0}\right)}\left|D^{2} f\left(D^{m} P\right)-D^{2} f\left(D^{m} P-t D^{m}(u-P)\right)\right|^{1 / 2} \mathrm{~d} t \\
& \times\left|D^{m}(u-P)\right| \mathrm{d} \mathcal{L}^{n} \sup _{B_{\rho}\left(x_{0}\right)}\left|D^{m} \varphi\right| \\
\leqslant & \left.\sqrt{2 L} f_{B_{\rho}\left(x_{0}\right)} \int_{0}^{1} v\left(\left|t\left(D^{m}(u-P)\right)\right|\right)^{1 / 2} \mathrm{~d} t \mid D^{m}(u-P)\right)\left|\mathrm{d} \mathcal{L}^{n} \sup _{B_{\rho}\left(x_{0}\right)}\right| D^{m} \varphi \mid \\
\leqslant & \sqrt{2 L}\left(f_{B_{\rho}\left(x_{0}\right)} v\left(\left|\left(D^{m}(u-P)\right)\right|\right) \mathrm{d} \mathcal{L}^{n}\right)^{1 / 2}\left(\underset{B_{\rho}\left(x_{0}\right)}{f}\left|D^{m}(u-P)\right|^{2} \mathrm{~d} \mathcal{L}^{n}\right)^{1 / 2} \\
& \times \sup _{B_{\rho}\left(x_{0}\right)}\left|D^{m} \varphi\right| \\
\leqslant & \sqrt{2 L} \sqrt{v\left(\Phi_{2}\left(x_{0}, \rho, P\right)\right)} \Phi_{2}\left(x_{0}, \rho, P\right) \sup _{B_{\rho}\left(x_{0}\right)}\left|D^{m} \varphi\right| .
\end{aligned}
$$

To prove (ii), we argue similarly using (H4), (15) and Remark 4

$$
\begin{aligned}
& \left|f_{B_{\rho}\left(x_{0}\right)} D^{2} f\left(D^{m} P\right)\left(D^{m}(u-P), D^{m} \varphi\right) \mathrm{d} \mathcal{L}^{n}\right| \\
& \leqslant f_{B_{\rho}\left(x_{0}\right)} \int_{0}^{1}\left|D^{2} f\left(D^{m} P\right)-D^{2} f\left(D^{m} P-t D^{m}(u-P)\right)\right|^{1 / p} \mathrm{~d} t \\
& \times\left(2^{p} L\left(1+M^{p-2}+\left|D^{m}(u-P)\right|^{p-2}\right)\right)^{\frac{p-1}{p}}\left|D^{m}(u-P)\right| \mathrm{d} \mathcal{L}^{n} \sup _{B_{\rho}\left(x_{0}\right)}\left|D^{m} \varphi\right| \\
& \leqslant f_{B_{\rho}\left(x_{0}\right)}\left(\left(1+M^{p-2}+\left|D^{m}(u-P)\right|^{p-2}\right) v_{M}\left(\left|D^{m}(u-P)\right|\right)\right)^{1 / p} \\
& \times\left(2^{p} L\left(1+M^{p-2}+\left|D^{m}(u-P)\right|^{p-2}\right)\right)^{\frac{p-1}{p}}\left|D^{m}(u-P)\right| \mathrm{d} \mathcal{L}^{n} \\
& \times \sup _{B_{\rho}\left(x_{0}\right)}\left|D^{m} \varphi\right| \\
& \leqslant 2^{p-1} L^{\frac{p-1}{p}}\left(1+M^{p-2}\right) \underset{B_{\rho}\left(x_{0}\right)}{f} v_{M}\left(\left|D^{m}(u-P)\right|\right)^{1 / p} \\
& \times\left(1+\left|D^{m}(u-P)\right|^{p-2}\right)\left|D^{m}(u-P)\right| \mathrm{d} \mathcal{L}^{n} \sup _{B_{\rho}\left(x_{0}\right)}\left|D^{m} \varphi\right| .
\end{aligned}
$$

Therefore Hölder's, Jensen's and Minkowski's inequality together with $\frac{p}{p-1} \leqslant 2$ imply

$$
\begin{aligned}
& \left|\underset{B_{\rho}\left(x_{0}\right)}{f} D^{2} f\left(D^{m} P\right)\left(D^{m}(u-P), D^{m} \varphi\right) \mathrm{d} \mathcal{L}^{n}\right| \\
& \quad \leqslant 2^{p-1} L^{\frac{p-1}{p}}\left(1+M^{p-2}\right) v_{M}\left(\Phi_{p}\left(x_{0}, \rho, P\right)\right)^{1 / p}
\end{aligned}
$$




$$
\begin{aligned}
& \times\left(\underset{B_{\rho}\left(x_{0}\right)}{f}\left(\left|D^{m}(u-P)\right|+\left|D^{m}(u-P)\right|^{p-1}\right)^{\frac{p}{p-1}} \mathrm{~d} \mathcal{L}^{n}\right)^{\frac{p-1}{p}} \sup _{B_{\rho}\left(x_{0}\right)}\left|D^{m} \varphi\right| \\
\leqslant & 2^{p-1} L^{\frac{p-1}{p}}\left(1+M^{p-2}\right) v_{M}\left(\Phi_{p}\left(x_{0}, \rho, P\right)\right)^{1 / p} \\
& \times\left({ }_{B_{\rho}\left(x_{0}\right)} 2^{\frac{1}{p-1}}\left(\left|D^{m}(u-P)\right|^{\frac{p}{p-1}}+\left|D^{m}(u-P)\right|^{p}\right) \mathrm{d} \mathcal{L}^{n}\right)^{\frac{p-1}{p}} \sup _{B_{\rho}\left(x_{0}\right)}\left|D^{m} \varphi\right| \\
\leqslant & 2^{p} L^{\frac{p-1}{p}}\left(1+M^{p-2}\right) v_{M}\left(\Phi_{p}\left(x_{0}, \rho, P\right)\right)^{1 / p} \\
& \times\left(\left({ }_{B_{\rho}\left(x_{0}\right)}\left|D^{m}(u-P)\right|^{2} \mathrm{~d} \mathcal{L}^{n}\right)^{1 / 2}+\left(f_{B_{\rho}\left(x_{0}\right)}\left|D^{m}(u-P)\right|^{p} \mathrm{~d} \mathcal{L}^{n}\right)^{\frac{p-1}{p}}\right) \sup _{B_{\rho}\left(x_{0}\right)}\left|D^{m} \varphi\right| \\
\leqslant & 2^{p+1} L^{\frac{p-1}{p}}\left(1+M^{p-2}\right) v_{M}\left(\Phi_{p}\left(x_{0}, \rho, P\right)\right)^{1 / p} \Phi_{p}\left(x_{0}, \rho, P\right) \sup _{B_{\rho}\left(x_{0}\right)}\left|D^{m} \varphi\right|,
\end{aligned}
$$

provided $\Phi_{p}\left(x_{0}, \rho, P\right) \leqslant 1$.

\section{Proof of Theorem 1}

\section{Step 1: Setting}

For $x_{0} \in \Omega$ and a given Ball $B_{\rho}\left(x_{0}\right) \Subset \Omega$ choose the unique polynomial $P_{x_{0}, \rho}$ of degree $m$ such that $D^{m-1} P_{x_{0}, \rho}$ minimizes

$$
\begin{gathered}
P \mapsto \int_{B_{\rho}\left(x_{0}\right)}\left|D^{m-1}(u-P)\right|^{2} \mathrm{~d} \mathcal{L}^{n} \quad \text { and } \\
\int_{B_{\rho}\left(x_{0}\right)} D^{k}\left(u-P_{x_{0}, \rho}\right) \mathrm{d} \mathcal{L}^{n}=0 \quad \text { for } 0 \leqslant k \leqslant m-2 .
\end{gathered}
$$

From (5) and (6) we see

$$
D^{m-1} P_{x_{0}, \rho}(x)=q_{x_{0}, \rho}+Q_{x_{0}, \rho}\left(x-x_{0}\right),
$$

where $q_{x_{0}, \rho}=\left(D^{m-1} u\right)_{x_{0}, \rho}$ and

$$
D^{m} P_{x_{0}, \rho} \equiv Q_{x_{0}, \rho}=\frac{n+2}{\rho^{2}} f_{B_{\rho}\left(x_{0}\right)} D^{m-1} u \otimes\left(x-x_{0}\right) \mathrm{d} \mathcal{L}^{n}(x) .
$$

With this choice of $P_{x_{0}, \rho}$ we write

$$
\begin{aligned}
& \Phi_{2}\left(x_{0}, \rho / 2, P_{x_{0}, \rho}\right)=\left(\underset{B_{\rho / 2}\left(x_{0}\right)}{f}\left|D^{m}\left(u-P_{x_{0}, \rho}\right)\right|^{2} \mathrm{~d} \mathcal{L}^{n}\right)^{1 / 2}, \\
& \Phi_{2}:=\Phi_{2}\left(x_{0}, \rho / 2, P_{x_{0}, \rho}\right),
\end{aligned}
$$




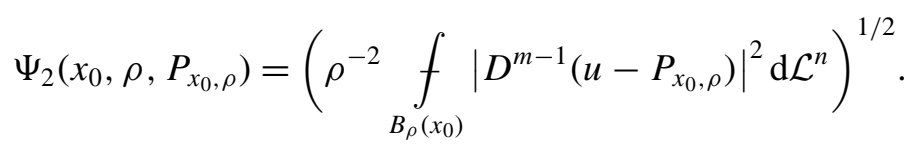

Since $\int_{B_{\rho}\left(x_{0}\right)} D^{k}\left(u-P_{x_{0}, \rho}\right) \mathrm{d} \mathcal{L}^{n}=0$ for $0 \leqslant k \leqslant m-1$ we can apply Caccioppoli's inequality Lemma 3 to infer

$$
\begin{aligned}
\Phi_{2}^{2} & =f_{B_{\rho / 2}\left(x_{0}\right)}\left|D^{m}\left(u-P_{x_{0}, \rho}\right)\right|^{2} \mathrm{~d} \mathcal{L}^{n} \leqslant C_{c a c}^{2} \rho^{-2} f_{B_{\rho}\left(x_{0}\right)}\left|D^{m-1}\left(u-P_{x_{0}, \rho}\right)\right|^{2} \mathrm{~d} \mathcal{L}^{n} \\
& =C_{c a c}^{2} \Psi_{2}^{2}\left(x_{0}, \rho, P_{x_{0}, \rho}\right) .
\end{aligned}
$$

Moreover, from Lemma 8(i) applied with $P=P_{x_{0}, \rho}$ on $B_{\rho / 2}\left(x_{0}\right)$ we see

$$
\left|f_{B_{\rho / 2}\left(x_{0}\right)} D^{2} f\left(D^{m} P_{x_{0}, \rho}\right)\left(D^{m}\left(u-P_{x_{0}, \rho}\right), D^{m} \varphi\right) \mathrm{d} \mathcal{L}^{n}\right| \leqslant C_{e u} \sqrt{v\left(\Phi_{2}\right)} \Phi_{2} \sup _{B_{\rho / 2}\left(x_{0}\right)}\left|D^{m} \varphi\right| .
$$

\section{Step 2: Decay estimate}

Claim 1. - For all $\theta \in] 0,1 / 4]$ there exists $\delta=\delta(n, N, \lambda, L, m, \theta)$ such that the smallness condition

$C_{e u} \sqrt{v\left(C_{c a c} \Psi_{2}\left(x_{0}, \rho, P_{x_{0}, \rho}\right)\right)}<\delta \quad$ implies $\quad \Psi_{2}^{2}\left(x_{0}, \rho, P_{x_{0}, \theta \rho}\right) \leqslant C_{d e c}^{2} \theta^{2} \Psi_{2}^{2}\left(x_{0}, \rho, P_{x_{0}, \rho}\right)$

with a constant $C_{d e c}=C_{d e c}(n, N, \lambda, L, m)$. Here $n, N, \lambda, L, m$ are as in hypotheses $(\mathrm{H} 1)$ and $(\mathrm{H} 2), v$ denotes the modulus of continuity of $D^{2} f$ (see Remark 4$), \Psi_{2}\left(x_{0}, \rho, P_{x_{0}, \rho}\right)$ is as in (32) and $C_{c a c}, C_{e u}$ are the constants from Lemma 3, Lemma 8(i) (see (33), (34)).

Proof. - We define

$$
v=\frac{u-P_{x_{0}, \rho}}{\Phi_{2}} \quad \text { with } \quad D^{m} v=\frac{D^{m}\left(u-P_{x_{0}, \rho}\right)}{\Phi_{2}} .
$$

In view of (34) this leads to

$$
\begin{gathered}
f_{B_{\rho / 2}\left(x_{0}\right)}\left|D^{m} v\right|^{2} \mathrm{~d} \mathcal{L}^{n}=\frac{\Phi_{2}^{2}}{\Phi_{2}^{2}}=1 \text { and } \\
\left|f_{B_{\rho / 2}\left(x_{0}\right)} D^{2} f\left(D^{m} P_{x_{0}, \rho}\right)\left(D^{m} v, D^{m} \varphi\right) \mathrm{d} \mathcal{L}^{n}\right| \leqslant C_{e u} \sqrt{v\left(\Phi_{2}\right)} \frac{\Phi_{2}}{\Phi_{2}} \sup _{B_{\rho / 2}\left(x_{0}\right)}\left|D^{m} \varphi\right| .
\end{gathered}
$$

For $\theta \in] 0,1 / 4]$ choose $\varepsilon=\theta^{n+4}$ and $\delta=\delta(n, N, \lambda, L, m, \varepsilon)=\delta(n, N, \lambda, L, m, \theta)$ from Lemma 6. Then $C_{e u} \sqrt{v\left(\Phi_{2}\right)} \leqslant C_{e u} \sqrt{v\left(C_{c a c} \Psi\left(x_{0}, \rho, P_{x_{0}, \rho}\right)\right)}<\delta$ by (33), our smallness assumption and the fact that $t \mapsto v(t)$ is non-decreasing. Hence the assumptions of Lemma 6 are fulfilled. This implies that there exists a $(\mathcal{A}, m)$-harmonic function $h$ with

$$
f_{B_{\rho / 2}\left(x_{0}\right)} D^{2} f\left(D^{m} P_{x_{0}, \rho}\right)\left(D^{m} h, D^{m} \varphi\right) \mathrm{d} \mathcal{L}^{n}=0,
$$




$$
\begin{gathered}
f_{B_{\rho / 2}\left(x_{0}\right)}\left|D^{m} h\right|^{2} \mathrm{~d} \mathcal{L}^{n} \leqslant 1 \quad \text { and } \\
\left(\frac{\rho}{2}\right)^{-2} f_{B_{\rho / 2}\left(x_{0}\right)}\left|D^{m-1} v-D^{m-1} h\right|^{2} \mathrm{~d} \mathcal{L}^{n} \leqslant \varepsilon=\theta^{n+4} .
\end{gathered}
$$

The standard a priori estimate for solutions of the linear system (36) (cf. Lemma 7) applied to $h$ on $B_{\rho / 2}\left(x_{0}\right)$ yields

$$
\begin{aligned}
\left(\frac{\rho}{2}\right)^{-2} \sup _{B_{\rho / 4}\left(x_{0}\right)}\left|D^{m} h\right|^{2}+\sup _{B_{\rho / 4}\left(x_{0}\right)}\left|D^{m+1} h\right|^{2} & \leqslant C_{\text {harm }}^{2}(\rho / 2)^{-2} f_{B_{\rho / 2}\left(x_{0}\right)}\left|D^{m} h\right|^{2} \mathrm{~d} \mathcal{L}^{n} \\
& \leqslant C_{\text {harm }}^{2}\left(\frac{\rho}{2}\right)^{-2} ;
\end{aligned}
$$

the last line follows from (37).

Applying Taylor's theorem to $h$ on $B_{\theta \rho}\left(x_{0}\right)$ we deduce

$$
\begin{aligned}
& \sup _{B_{\theta \rho}\left(x_{0}\right)}\left|D^{m-1} h(x)-D^{m-1} h\left(x_{0}\right)-D^{m} h\left(x_{0}\right)\left(x-x_{0}\right)\right|^{2} \\
& \leqslant\left(\frac{(\theta \rho)^{2}}{2 !} \sup _{B_{\rho / 4}\left(x_{0}\right)}\left|D^{m+1} h\right|\right)^{2} \leqslant C_{\text {harm }}^{2} \theta^{4} \rho^{2} .
\end{aligned}
$$

Denotion by $P_{x_{0}, \theta \rho}$ the unique polynomial associated to $u$ on $B_{\theta \rho}\left(x_{0}\right)$ by the construction from Step 1, we infer using the minimal property (28) of $P_{x_{0}, \theta \rho}$, the above mentioned a priori estimate for $h$ and Caccioppoli's inequality (33):

$$
\begin{aligned}
\theta \rho & \Psi_{2}\left(x_{0}, \theta \rho, P_{x_{0}, \theta \rho}\right) \\
= & \left(f_{B_{\theta \rho}\left(x_{0}\right)}\left|D^{m-1}\left(u-P_{x_{0}, \theta \rho}\right)\right|^{2} \mathrm{~d} \mathcal{L}^{n}\right)^{1 / 2} \\
\leqslant & \left(f_{B_{\theta \rho}\left(x_{0}\right)} \mid D^{m-1} u-D^{m-1} P_{x_{0}, \rho}\right. \\
& \left.-\left.\Phi_{2}\left(D^{m-1} h\left(x_{0}\right)+D^{m} h\left(x_{0}\right)\left(x-x_{0}\right)\right)\right|^{2} \mathrm{~d} \mathcal{L}^{n}\right)^{1 / 2} \\
\leqslant & \left(\underset{B_{\theta \rho}\left(x_{0}\right)}{f}\left|D^{m-1} v-\left(D^{m-1} h\left(x_{0}\right)+D^{m} h\left(x_{0}\right)\left(x-x_{0}\right)\right)\right|^{2} \mathrm{~d} \mathcal{L}^{n}\right)^{1 / 2} \Phi_{2} \\
\leqslant & \left(\underset{B_{\theta \rho}\left(x_{0}\right)}{f}\left|D^{m-1} v-D^{m-1} h\right|^{2} \mathrm{~d} \mathcal{L}^{n}\right)^{1 / 2} \Phi_{2} \\
& +\left(\underset{B_{\theta \rho}\left(x_{0}\right)}{f}\left|D^{m-1} h-D^{m-1} h\left(x_{0}\right)-D^{m} h\left(x_{0}\right)\left(x-x_{0}\right)\right|^{2} \mathrm{~d} \mathcal{L}^{n}\right)^{1 / 2} \Phi_{2}
\end{aligned}
$$




$$
\leqslant\left(2^{-\frac{n+2}{2}}+C_{h a r m}\right) \theta^{2} \rho \Phi_{2} \leqslant C_{d e c} \theta^{2} \rho \Psi_{2}\left(x_{0}, \rho, P_{x_{0}, \rho}\right)
$$

with $C_{d e c}=\left(2^{-\frac{n+2}{2}}+C_{\text {harm }}\right) C_{c a c}$. This proves Claim 1 .

Given $0<\alpha<1$ we now fix $\theta$ sufficiently small such that $C_{d e c} \theta \leqslant \theta^{\alpha}$. This fixes $\varepsilon=$ $\theta^{n+4}$ and also $\delta=\delta(n, N, \lambda, L, m, \varepsilon)$. We choose $s_{0}>0$ such that $C_{e u} \sqrt{\left(v\left(C_{c a c} s_{0}\right)\right)}<\delta$. Then, if for some ball $B_{\rho}\left(x_{0}\right) \Subset \Omega$ the smallness condition $\Psi_{2}\left(x_{0}, \rho, P_{x_{0}, \rho}\right) \leqslant s_{0}$ is satisfied, we have the decay estimate

$$
\Psi_{2}^{2}\left(x_{0}, \theta \rho, P_{x_{0}, \theta \rho}\right) \leqslant \theta^{2 \alpha} \Psi_{2}^{2}\left(x_{0}, \rho, P_{x_{0}, \rho}\right) .
$$

\section{Step 3: Iteration}

Claim 2. - Suppose $\Psi_{2}\left(x_{0}, \rho, P_{x_{0}, \rho}\right) \leqslant s_{0}$ for some ball $B_{\rho}\left(x_{0}\right) \Subset \Omega$. Then for all $k \in \mathbb{N}$

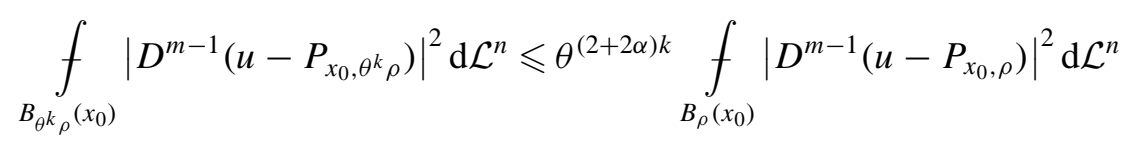

i.e., $\Psi_{2}\left(x_{0}, \theta^{k} \rho, P_{x_{0}, \theta^{k} \rho}\right) \leqslant \theta^{(2+2 \alpha) k} \Psi_{2}\left(x_{0}, \rho, P_{x_{0}, \rho}\right)$.

Proof. - For $k=1$ this is still the decay estimate (40). By induction we see, noting that the smallness condition $\Psi_{2}\left(x_{0}, \theta^{l} \rho, P_{x_{0}, \theta^{l} \rho}\right) \leqslant s_{0}$ can be verified iteratively for $l=1, \ldots, k-1$, that

$$
\begin{aligned}
& \underset{B_{\theta^{k}}\left(x_{0}\right)}{f}\left|D^{m-1}\left(u-P_{x_{0}, \theta^{k} \rho}\right)\right|^{2} \mathrm{~d} \mathcal{L}^{n} \leqslant \theta^{(2+2 \alpha)} f_{B_{\theta^{k-1}}\left(x_{0}\right)}\left|D^{m-1}\left(u-P_{x_{0}, \theta^{k-1} \rho}\right)\right|^{2} \mathrm{~d} \mathcal{L}^{n} \\
& \leqslant \theta^{(2+2 \alpha) k} f_{B_{\rho}\left(x_{0}\right)}\left|D^{m-1}\left(u-P_{x_{0}, \rho}\right)\right|^{2} \mathrm{~d} \mathcal{L}^{n} .
\end{aligned}
$$

This proves the asserted estimate.

\section{Step 4: Partial regularity}

For $0<r<\rho$ we fix $k \in \mathbb{N}_{0}$ with $\theta^{k+1} \rho<r \leqslant \theta^{k} \rho$. Then Step 3 implies

$$
\begin{aligned}
f_{B_{r}\left(x_{0}\right)}\left|D^{m-1}\left(u-P_{x_{0}, r}\right)\right|^{2} \mathrm{~d} \mathcal{L}^{n} & \leqslant f_{B_{r}\left(x_{0}\right)}\left|D^{m-1}\left(u-P_{x_{0}, \theta^{k} \rho}\right)\right|^{2} \mathrm{~d} \mathcal{L}^{n} \\
& \leqslant \theta^{-(n+2+2 \alpha)}\left(\frac{r}{\rho}\right)^{2+2 \alpha} \underset{B_{\rho}\left(x_{0}\right)}{f_{\mid}\left|D^{m-1}\left(u-P_{x_{0}, \rho}\right)\right|^{2} \mathrm{~d} \mathcal{L}^{n} .}
\end{aligned}
$$

Using Poincaré's inequality Lemma 1 with $k=0, l=m-1$ on $B_{r}\left(x_{0}\right)$ (which is possible by our choice of $P_{x_{0}, r}$ ) we obtain

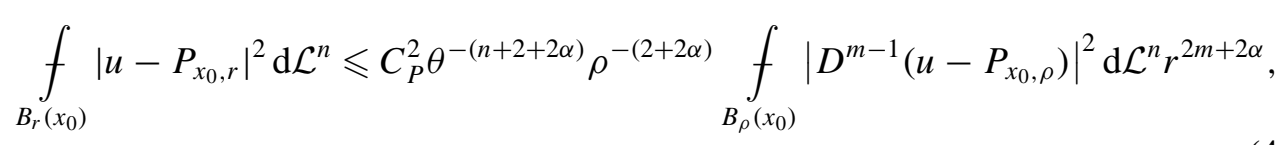


provided we have $\Psi_{2}\left(x_{0}, \rho, P_{x_{0}, \rho}\right)<s_{0}$.

Suppose $x_{0} \in \Omega \backslash \Sigma$. Then $\liminf _{\rho \searrow 0} f_{B_{\rho}\left(x_{0}\right)}\left|D u-\left(D^{m} u\right)_{x_{0}, \rho}\right|^{2}=0$ and therefore (using Poincaré's inequality and recalling the choice of $P_{x_{0}, \rho}$ )

$$
\begin{aligned}
\Psi_{2}^{2}\left(x_{0}, \rho, P_{x_{0}, \rho}\right) & =\rho^{-2} f_{B_{\rho}\left(x_{0}\right)}\left|D^{m-1}\left(u-P_{x_{0}, \rho}\right)\right|^{2} \mathrm{~d} \mathcal{L}^{n} \\
& \leqslant \rho^{-2} f_{B_{\rho}\left(x_{0}\right)}\left|D^{m-1} u-\left(D^{m-1} u\right)_{x_{0}, \rho}-\left(D^{m} u\right)_{x_{0}, \rho}\left(x-x_{0}\right)\right|^{2} \mathrm{~d} \mathcal{L}^{n} \\
& \leqslant C_{p}^{2} f_{B_{\rho}\left(x_{0}\right)}\left|D^{m} u-\left(D^{m} u\right)_{x_{0}, \rho}\right|^{2} \mathrm{~d} \mathcal{L}^{n} .
\end{aligned}
$$

Hence

$$
\left(\underset{B_{\rho}\left(x_{0}\right)}{f}\left|D^{m} u-\left(D^{m} u\right)_{x_{0}, \rho}\right|^{2} \mathrm{~d} \mathcal{L}^{n}\right)^{1 / 2}<\frac{s_{0}}{C_{p}}
$$

implies $\Psi_{2}\left(x_{0}, \rho, P_{x_{0}, \rho}\right)<s_{0}$. Since $y \mapsto f_{B_{\rho}(y)}\left|D^{m} u-\left(D^{m} u\right)_{y, \rho}\right|^{2} \mathrm{~d} \mathcal{L}^{n}$ is continuous, we see that (43) implies the same condition for any $y \in B_{\sigma}\left(x_{0}\right), \sigma>0$. Therefore $\Psi_{2}\left(y, \rho, P_{y, \rho}\right)<s_{0}$ for any $y \in B_{\sigma}\left(x_{0}\right)$ and (41) holds for $y \in B_{\sigma}\left(x_{0}\right)$ and any $0<r<\rho$. Campanato's integral characterization (4) of $C^{m, \alpha}$ functions shows $C^{m, \alpha}$-regularity of $u$ on $B_{\sigma / 2}\left(x_{0}\right)$. Finishing the proof of Theorem 1 we note, that $\mathcal{L}^{n}(\Sigma)=0$ by the Lebesgue differentiation theorem.

\section{Proof of Theorem 2}

\section{Step 1: Setting}

In this section we modify the arguments of the last section, starting with the same setting:

For a given ball $B_{\rho}\left(x_{0}\right)$ choose the unique polynomial $P_{x_{0}, \rho}$ of degree $m$ specified in (28) and (29). Let

$$
\begin{gathered}
\Phi_{p}\left(x_{0}, \rho / 2, P_{x_{0}, \rho}\right)=\left(f_{B_{\rho / 2}\left(x_{0}\right)}\left|D^{m}\left(u-P_{x_{0}, \rho}\right)\right|^{2} \mathrm{~d} \mathcal{L}^{n}+f_{B_{\rho / 2}\left(x_{0}\right)}\left|D^{m}\left(u-P_{x_{0}, \rho}\right)\right|^{p} \mathrm{~d} \mathcal{L}^{n}\right)^{1 / 2}, \\
\Psi_{p}\left(x_{0}, \rho, P_{x_{0}, \rho}\right) \\
=\left(\rho^{-2} f_{B_{\rho}\left(x_{0}\right)}\left|D^{m-1}\left(u-P_{x_{0}, \rho}\right)\right|^{2} \mathrm{~d} \mathcal{L}^{n}+\rho^{-p} f_{B_{\rho}\left(x_{0}\right)}\left|D^{m-1}\left(u-P_{x_{0}, \rho}\right)\right|^{p} \mathrm{~d} \mathcal{L}^{n}\right)^{1 / 2}, \\
\Phi_{p}:=\Phi_{p}\left(x_{0}, \rho / 2, P_{x_{0}, \rho}\right), \quad \Psi_{p}:=\Psi_{p}\left(x_{0}, \rho, P_{x_{0}, \rho}\right) .
\end{gathered}
$$

From Lemmas 5 and 8 with $\varphi \in C_{0}^{\infty}\left(B_{\rho / 2}\left(x_{0}\right), R^{N}\right)$ we see 


$$
\begin{aligned}
& \Phi_{p}^{2}=\underset{B_{\rho / 2}\left(x_{0}\right)}{f}\left|D^{m}\left(u-P_{x_{0}, \rho}\right)\right|^{2} \mathrm{~d} \mathcal{L}^{n}+\underset{B_{\rho / 2}\left(x_{0}\right)}{f}\left|D^{m}\left(u-P_{x_{0}, \rho}\right)\right|^{p} \mathrm{~d} \mathcal{L}^{n} \\
& \leqslant C_{c a c}^{2}\left(\rho^{-2} f_{B_{\rho}\left(x_{0}\right)}\left|D^{m-1}\left(u-P_{x_{0}, \rho}\right)\right|^{2} \mathrm{~d} \mathcal{L}^{n}+\rho^{-p} f D_{B_{\rho}\left(x_{0}\right)}\left|D^{m-1}\left(u-P_{x_{0}, \rho}\right)\right|^{p} \mathrm{~d} \mathcal{L}^{n}\right) \\
&=C_{c a c}^{2} \Psi_{p}^{2}, \quad \\
& \underset{B_{\rho / 2}\left(x_{0}\right)}{f} D^{2} f\left(D^{m} P_{x_{0}, \rho}\right)\left(D^{m}\left(u-P_{x_{0}, \rho}\right), D^{m} \varphi\right) \mathrm{d} \mathcal{L}^{n} \mid \\
& \leqslant C_{e u} v_{M}\left(\Phi_{p}\right)^{1 / p} \Phi_{p} \sup _{B_{\rho / 2}\left(x_{0}\right)}\left|D^{m} \varphi\right|,
\end{aligned}
$$

provided $\left|D^{m} P_{x_{0}, \rho}\right| \leqslant M$. Note, that in difference to (33) and (34) the constants $C_{c a c}=$ $C_{c a c}(M)$ and $C_{e u}=C_{e u}(M)$ depend on $M$.

\section{Step 2: Decay estimate}

Claim 1. - Let $M>0$ fixed. For any $\theta \in] 0,1 / 4]$ there exists $\delta=\delta(n, N, \lambda, L, m, \theta)$ and $q=q(n, p)$ such that if

(1) $C_{e u}(M) v_{M}\left(C_{c a c}(M) \Psi_{p}\left(x_{0}, \rho, P_{x_{0}, \rho}\right)\right)^{1 / p}<\delta$,

(2) $\Psi_{p}\left(x_{0}, \rho, P_{x_{0}, \rho}\right) \leqslant \theta^{q}$, and

(3) $\left|D^{m} P_{x_{0}, \rho}\right| \leqslant M$,

then

$$
\Psi_{p}^{2}\left(x_{0}, \theta \rho, P_{x_{0}, \theta \rho}\right) \leqslant C_{d e c}^{2} \theta^{2} \Psi_{p}^{2}\left(x_{0}, \rho, P_{x_{0}, \rho}\right)
$$

with a constant $C_{d e c}(M)=C_{d e c}(n, N, \lambda, L, m, M)$. Here $n, N, \lambda, L, m$ are as in hypotheses $(\mathrm{H} 3)$ and $(\mathrm{H} 4), v_{M}$ is the modulus of continuity for $D^{2} f$ from Remark 4, $\Psi_{p}$ is defined in Step 1 and the constants $C_{c a c}(M), C_{e u}(M)$ are from Lemma 5 and Lemma 8(ii).

Proof. - As in the proof of Theorem 1 we define

$$
v=\frac{\left(u-P_{x_{0}, \rho}\right)}{\Phi_{p}} \quad \text { with } \quad D^{m} v=\frac{D^{m}\left(u-P_{x_{0}, \rho}\right)}{\Phi_{p}}
$$

In view of (45) and hypotheses (1) and (3) we can make the same observations as in the proof of theorem $(1)$ in regards to an application of the $(\mathcal{A}, m)$-harmonic approximation lemma. Arguing in the same way we get the same conclusions as in the $(p=2)$ case, i.e. we get the estimate (39)

$$
\underset{B_{\theta \rho}\left(x_{0}\right)}{f}\left|D^{m-1}\left(u-P_{\theta x_{0}, \rho}\right)\right|^{2} \mathrm{~d} \mathcal{L}^{n} \leqslant\left(2^{-\frac{n+2}{2}}+C_{h a r m}\right)^{2}\left(\theta^{2} \rho\right)^{2} \Phi_{p}^{2}=: C_{1}^{2}\left(\theta^{2} \rho\right)^{2} \Phi_{p}^{2}
$$

for $\theta \in] 0,1 / 4]$, provided $C_{e u} v_{M}\left(\Phi_{p}\right)^{1 / p} \leqslant \delta=\delta(n, N, \lambda, L, m, \theta)$. 
Next we derive an estimate for the second term $f_{B_{\theta_{\rho}}\left(x_{0}\right)}\left|D^{m-1}\left(u-P_{x_{0}, \theta \rho}\right)\right|^{p} \mathrm{~d} \mathcal{L}^{n}$ of the right side in Caccioppoli's inequality. ${ }^{3}$ For this we let (in the case $p>2$ )

$$
p^{*}= \begin{cases}\frac{n p}{n-p}>p, & \text { the conjugated Sobolev exponent to } p \text { in the case } 2<p<n, \\ p^{*}>p, & \text { fixed in the case } p \geqslant n,\end{cases}
$$

with $\frac{1}{2}>\frac{1}{p}>\frac{1}{p^{*}}$. Therefore we find $\left.t \in\right] 0,1[$ such that

$$
\frac{1}{p}=(1-t) \frac{1}{2}+t \frac{1}{p^{*}}
$$

Using Hölder's inequality, Sobolev's inequality (9), Caccioppolli’s inequality (44) and (47) we compute:

$$
\begin{aligned}
& \underset{B_{\theta \rho}\left(x_{0}\right)}{f}\left|D^{m-1}\left(u-P_{x_{0}, \theta \rho}\right)\right|^{p} \mathrm{~d} \mathcal{L}^{n} \\
& \leqslant\left(f_{B_{\theta \rho}\left(x_{0}\right)}\left|D^{m-1}\left(u-P_{x_{0}, \theta \rho}\right)\right|^{2} \mathrm{~d} \mathcal{L}^{n}\right)^{(1-t) \frac{p}{2}}\left(f_{B_{\theta \rho}\left(x_{0}\right)}\left|D^{m-1}\left(u-P_{x_{0}, \theta \rho}\right)\right|^{p^{*}} \mathrm{~d} \mathcal{L}^{n}\right)^{t \frac{p}{p^{*}}} \\
& \leqslant C_{1}^{(1-t) p}\left(\theta^{2} \rho\right)^{(1-t) p} \Phi_{p}^{(1-t) p} C_{S}^{t p}(\theta \rho)^{t p}\left(f_{B_{\theta \rho}\left(x_{0}\right)}\left|D^{m}\left(u-P_{x_{0}, \theta \rho}\right)\right|^{p} \mathrm{~d} \mathcal{L}^{n}\right)^{t} \\
& \leqslant C_{2}^{p} \mathcal{L}^{n}\left(B_{\theta \rho}\left(x_{0}\right)\right)^{-t}(\theta \rho)^{p} \theta^{(1-t) p} \Psi_{p}^{(1-t) p}\left(\int_{B_{\theta \rho}\left(x_{0}\right)}\left|D^{m}\left(u-P_{x_{0}, \theta \rho}\right)\right|^{p} \mathrm{~d} \mathcal{L}^{n}\right)^{t}
\end{aligned}
$$

where $C_{2}=C_{c a c}^{(1-t)} C_{1}^{(1-t)} C_{S}^{t}=C_{2}\left(n, N, \lambda, L, m, M, p, p^{*}\right)$. From Lemma 2 we deduce $\left(P_{x_{0}, \theta \rho}\right.$ denoting again the unique polynomial associated to $u$ on $\left.B_{\theta \rho}\left(x_{0}\right)\right)$

$$
\begin{aligned}
& \left(\int_{B_{\theta \rho}\left(x_{0}\right)}\left|D^{m}\left(u-P_{x_{0}, \theta \rho}\right)\right|^{p} \mathrm{~d} \mathcal{L}^{n}\right)^{1 / p} \\
& \leqslant\left(\int_{B_{\theta \rho}\left(x_{0}\right)}\left|D^{m}\left(u-P_{x_{0}, \rho}\right)\right|^{p} \mathrm{~d} \mathcal{L}^{n}\right)^{1 / p}+\mathcal{L}^{n}\left(B_{\theta \rho}\left(x_{0}\right)\right)^{1 / p}\left|D^{m} P_{x_{0}, \theta \rho}-D^{m} P_{x_{0}, \rho}\right| \\
& \leqslant \mathcal{L}^{n}\left(B_{\rho / 2}\left(x_{0}\right)\right)^{1 / p}\left(f_{B_{\rho / 2}\left(x_{0}\right)}\left|D^{m}\left(u-P_{x_{0}, \rho}\right)\right|^{p} \mathrm{~d} \mathcal{L}^{n}\right)^{1 / p} \\
& \quad+\mathcal{L}^{n}\left(B_{\theta \rho}\left(x_{0}\right)\right)^{1 / p}\left(n(n+2)(\theta \rho)^{-2} \underset{B_{\theta \rho}\left(x_{0}\right)}{f}\left|D^{m-1}\left(u-P_{x_{0}, \rho}\right)\right|^{2} \mathrm{~d} \mathcal{L}^{n}\right)^{1 / 2}
\end{aligned}
$$

\footnotetext{
${ }^{3}$ Observe, that by Campanato's integral characterization, in regular points of $u$, this term is much smaller than $f_{B_{\theta \rho}\left(x_{0}\right)}\left|D^{m-1}\left(u-P_{x_{0}, \rho}\right)\right|^{2} \mathrm{~d} \mathcal{L}^{n}$.
} 


$$
\begin{aligned}
& \leqslant\left(\mathcal{L}^{n}\left(B_{\rho / 2}\left(x_{0}\right)\right) C_{c a c}^{2} \Psi_{p}^{2}\right)^{1 / p}+\sqrt{n(n+2)}(\theta \rho)^{-1}\left(\int_{B_{\rho}\left(x_{0}\right)}\left|D^{m-1}\left(u-P_{x_{0}, \rho}\right)\right|^{p} \mathrm{~d} \mathcal{L}^{n}\right)^{1 / p} \\
& \leqslant\left(\mathcal{L}^{n}\left(B_{\rho}\left(x_{0}\right)\right)\right)^{1 / p} \theta^{-1}\left(\left(2^{-n} C_{c a c}^{2}\right)^{1 / p}+\sqrt{n(n+2)}\right) \Psi_{p}^{2 / p} .
\end{aligned}
$$

From this estimate, inserted in inequality (49) and Young's inequality we get, letting $C_{3}^{2}=C_{2}^{p}\left(\left(2^{-n} C_{c a c}^{2}\right)^{1 / p}+\sqrt{n(n+2)}\right)^{t p}=C_{3}^{2}\left(n, N, \lambda, L, m, M, p, p^{*}\right)$

$$
\begin{aligned}
(\theta \rho)^{-p} f_{B_{\theta \rho}\left(x_{0}\right)}\left|D^{m-1}\left(u-P_{x_{0}, \rho}\right)\right|^{p} \mathrm{~d} \mathcal{L}^{n} & \leqslant C_{3}^{2} \theta^{(1-t) p} \Psi_{p}^{(1-t) p} \theta^{-(n+p) t} \Psi_{p}^{2 t} \\
& \leqslant C_{3}^{2}\left(\frac{(1-t) p}{2} \theta^{2} \Psi_{p}^{2}+\frac{t p}{p^{*}} \theta^{-\frac{(n+p) p^{*}}{p}} \Psi_{p}^{2 p^{*} / p}\right) \\
& \leqslant C_{3}^{2} \theta^{2} \Psi_{p}^{2}
\end{aligned}
$$

provided $\Psi_{p}^{2\left(p^{*}-p\right) / p} \leqslant \theta^{(n+p) p^{*} / p+2}$. If we choose $q=\frac{(n+p) p^{*}+2 p}{2\left(p^{*}-p\right)}$ in hypothesis (2) the desired estimate follows from (44), (47) and (50) with $C_{d e c}^{2}=C_{1}^{2} C_{c a c}^{2}+C_{3}^{2}$. Note that $C_{d e c}=C_{d e c}(M)$.

Given $0<\alpha<1$ we now fix $\theta$ sufficiently small such that $C_{d e c}(2 M) \theta \leqslant \theta^{\alpha}$. This also fixes $\varepsilon=\theta^{n+4}$ and $\delta=\delta(n, N, \lambda, L, m, \varepsilon)$. We choose $s_{0}>0$ (depending on $2 M$ also) such that $C_{e u}(2 M)\left(v_{2 M}\left(C_{c a c}(2 M) s_{0}\right)^{1 / p}<\delta\right.$. Then, if for some ball $B_{\rho}\left(x_{0}\right) \Subset \Omega$ the conditions

$$
\Psi_{p}\left(x_{0}, \rho, P_{x_{0}, \rho}\right) \leqslant s_{0}, \quad \Psi_{p}\left(x_{0}, \rho, P_{x_{0}, \rho}\right) \leqslant \theta^{q} \quad \text { and } \quad\left|D^{m} P_{x_{0}, \rho}\right| \leqslant 2 M
$$

are satisfied, we have the decay estimate

$$
\Psi_{p}^{2}\left(x_{0}, \theta \rho, P_{x_{0}, \theta \rho}\right) \leqslant \theta^{2 \alpha} \Psi_{p}^{2}\left(x_{0}, \rho, P_{x_{0}, \rho}\right) .
$$

\section{Step 3: Iteration}

CLAIM 2. - Suppose $\Psi_{p}\left(x_{0}, \rho, P_{x_{0}, \rho}\right) \leqslant s_{0}, \Psi_{p}\left(x_{0}, \rho, P_{x_{0}, \rho}\right) \leqslant \theta^{q}$ and $\left|D^{m} P_{x_{0}, \rho}\right| \leqslant$ $M$ for some ball $B_{\rho}\left(x_{0}\right) \Subset \Omega$. Suppose further that

$$
\Psi_{p}\left(x_{0}, \rho, P_{x_{0}, \rho}\right) \leqslant \frac{\theta^{\frac{n+2}{2}}\left(1-\theta^{\alpha}\right)}{\sqrt{n(n+2)}} M .
$$

Then for all $k \in \mathbb{N}$

$$
\Psi_{p}^{2}\left(x_{0}, \theta^{k} \rho, P_{x_{0}, \theta^{k} \rho}\right) \leqslant \theta^{(2+2 \alpha) k} \Psi_{p}^{2}\left(x_{0}, \rho, P_{x_{0}, \rho}\right) .
$$

Proof. - For $k=1$ this follows from the decay estimate (52). Suppose that the statement is true for all $l \leqslant k-1 \in \mathbb{N}_{0}$. Firstly, we will show that the assumptions (51) of the decay estimate (52) are fulfilled on the ball $B_{\theta^{k} \rho}\left(x_{0}\right)$. Therefore it remains to show, that $\left|D^{m} P_{x_{0}, \theta^{k}}\right| \leqslant 2 M$. From (30) and Lemma 2 we infer 


$$
\begin{aligned}
\left|D^{m} P_{x_{0}, \theta^{k}}\right| & \leqslant M+\sum_{j=0}^{k-1}\left|D^{m} P_{x_{0}, \theta^{j+1} \rho}-D^{m} P_{x_{0}, \theta^{j} \rho}\right| \\
& \leqslant M+\sqrt{n(n+2)} \theta^{-(n+2) / 2} \sum_{j=0}^{k-1} \frac{1}{\theta^{j} \rho}\left(f_{B_{\theta^{j}}\left(x_{0}\right)}\left|D^{m-1}\left(u-P_{x_{0}, \theta^{j} \rho}\right)\right|^{2} \mathrm{~d} \mathcal{L}^{n}\right)^{1 / 2} \\
& =M+\sqrt{n(n+2)} \theta^{-(n+2) / 2} \sum_{j=0}^{k-1} \Psi_{p}\left(x_{0}, \theta^{j} \rho, P_{x_{0}, \theta^{j} \rho}\right) \\
& \leqslant M+\sqrt{n(n+2)} \theta^{-(n+2) / 2} \sum_{j=0}^{k-1} \theta^{\alpha j} \Psi_{p}\left(x_{0}, \rho, P_{x_{0}, \rho}\right) \\
& \leqslant M+\sqrt{n(n+2)} \theta^{-(n+2) / 2} \frac{1}{1-\theta^{\alpha}} \Psi_{p}\left(x_{0}, \rho, P_{x_{0}, \rho}\right) \\
& \leqslant 2 M
\end{aligned}
$$

provided $\Psi_{p}\left(x_{0}, \rho, P_{x_{0}, \rho}\right) \leqslant \theta^{\frac{n+2}{2}}\left(1-\theta^{\alpha}\right) / \sqrt{n(n+2)} M$. Hence we can apply (52) and conclude the estimate at level $k$.

\section{Step 4: Partial regularity}

For $0<r<\rho$ fix $k \in \mathbb{N}_{0}$ with $\theta^{k+1} \rho<r \leqslant \theta^{k} \rho$. Then Step 3 implies

$$
\begin{aligned}
& f_{B_{r}\left(x_{0}\right)}\left|D^{m-1}\left(u-P_{x_{0}, r}\right)\right|^{2} \mathrm{~d} \mathcal{L}^{n} \leqslant \frac{\left(\theta^{k} \rho\right)^{n}}{r^{n}} \underset{B_{\theta^{k}}\left(x_{0}\right)}{f}\left|D^{m-1}\left(u-P_{x_{0}, \theta^{k} \rho}\right)\right|^{2} \mathrm{~d} \mathcal{L}^{n} \\
& \leqslant \frac{\left(\theta^{k} \rho\right)^{n}}{r^{n}}\left(\theta^{k} \rho\right)^{2} \Psi_{p}^{2}\left(x_{0}, \theta^{k} \rho, P_{x_{0}, \theta^{k} \rho}\right) \leqslant \frac{\left(\theta^{k} \rho\right)^{n}}{r^{n}} \theta^{(2+2 \alpha) k} \rho^{2} \Psi_{p}^{2}\left(x_{0}, \rho, P_{x_{0}, \rho}\right) \\
& \leqslant \theta^{-(n+2+2 \alpha)}\left(\frac{r}{\rho}\right)^{(2+2 \alpha)} \rho^{2} \Psi_{p}^{2}\left(x_{0}, \rho, P_{x_{0}, \rho}\right) .
\end{aligned}
$$

Using Poincaré's inequality Lemma 1 with $k=0, l=m-1$ on $B_{r}\left(x_{0}\right)$ we obtain

$$
\underset{B_{r}\left(x_{0}\right)}{f}\left|u-P_{x_{0}, r}\right|^{2} \mathrm{~d} \mathcal{L}^{n} \leqslant C_{P}^{2} \theta^{-(n+2+2 \alpha)} \rho^{-2 \alpha} \Psi_{p}^{2}\left(x_{0}, \rho, P_{x_{0}, \rho}\right) r^{2 m+2 \alpha}
$$

provided

$$
\Psi_{p}\left(x_{0}, \rho, P_{x_{0}, \rho}\right)<\max \left(s_{0}, \theta^{q}, \frac{\theta^{\frac{n+2}{2}}\left(1-\theta^{\alpha}\right)}{\sqrt{n(n+2)}} M\right)=: S_{0}
$$

and $\left|D^{m} P_{x_{0}, \rho}\right|<M$.

Suppose $x_{0} \in \Omega \backslash\left(\Sigma_{1} \cup \Sigma_{2}\right)$, where $\Sigma_{1}$ and $\Sigma_{2}$ are from the formulation of Theorem 2 . Then $\liminf _{\rho \searrow 0} f_{B_{\rho}\left(x_{0}\right)}\left|D u-\left(D^{m} u\right)_{x_{0}, \rho}\right|^{2}=0$ and $\lim \sup _{\rho \searrow 0}\left(D^{m} u\right)_{x_{0}, \rho}<\infty$. 
Using Lemma 2(ii) and Hölder's inequality we see

$$
\left|D^{m} P_{x_{0}, \rho}\right| \leqslant\left|\left(D^{m} u\right)_{x_{0}, \rho}\right|+C_{p} \sqrt{n(n+2)}\left(f_{B_{\rho}\left(x_{0}\right)}\left|D^{m} u-\left(D^{m} u\right)_{x_{0}, \rho}\right|^{p} \mathrm{~d} \mathcal{L}^{n}\right)^{1 / p}
$$

Hence

$$
\begin{aligned}
& \left|\left(D^{m} u\right)_{x_{0}, \rho}\right|<\frac{M}{2} \text { and } \\
& \quad\left(\underset{B_{\rho}\left(x_{0}\right)}{f}\left|D^{m} u-\left(D^{m} u\right)_{x_{0}, \rho}\right|^{p} \mathrm{~d} \mathcal{L}^{n}\right)^{1 / p}<\frac{M}{2 C_{p} \sqrt{n(n+2)}}
\end{aligned}
$$

implies $\left|D^{m} P_{x_{0}, \rho}\right|<M$.

Next we will show that the smallness conditions for $\Psi_{p}\left(x_{0}, \rho, P_{x_{0}, \rho}\right)$ can be achieved on $\Omega \backslash\left(\Sigma_{1} \cup \Sigma_{2}\right)$. Firstly, we see from (42) and Hölder's inequality

$$
\begin{aligned}
\rho^{-2} f_{B_{\rho}\left(x_{0}\right)}\left|D^{m-1}\left(u-P_{x_{0}, \rho}\right)\right|^{2} \mathrm{~d} \mathcal{L}^{n} & \leqslant C_{p}^{2} f_{B_{\rho}\left(x_{0}\right)}\left|D^{m} u-\left(D^{m} u\right)_{x_{0}, \rho}\right|^{2} \mathrm{~d} \mathcal{L}^{n} \\
& \leqslant C_{p}^{2}\left(\underset{B_{\rho}\left(x_{0}\right)}{f}\left|D^{m} u-\left(D^{m} u\right)_{x_{0}, \rho}\right|^{p} \mathrm{~d} \mathcal{L}^{n}\right)^{2 / p} .
\end{aligned}
$$

Secondly, we have from (7), Lemma 2(ii) and the above estimate

$$
\begin{aligned}
& \rho^{-p} \underset{B_{\rho}\left(x_{0}\right)}{f}\left|D^{m-1}\left(u-P_{x_{0}, \rho}\right)\right|^{p} \mathrm{~d} \mathcal{L}^{n} \\
& \leqslant C_{p}^{p} f_{B_{\rho}\left(x_{0}\right)}\left|D^{m}\left(u-P_{x_{0}, \rho}\right)\right|^{p} \mathrm{~d} \mathcal{L}^{n} \\
& \leqslant C_{p}^{p}\left[\left(\int_{B_{\rho}\left(x_{0}\right)}\left|D^{m} u-\left(D^{m} u\right)_{x_{0}, \rho}\right|^{p} \mathrm{~d} \mathcal{L}^{n}\right)^{1 / p}+\left|D^{m} P_{x_{0}, \rho}-\left(D^{m} u\right)_{x_{0}, \rho}\right|\right]^{p} \\
& \leqslant C_{p}^{p}\left(1+C_{p} \sqrt{n(n+2)}\right)^{p} \underset{B_{\rho}\left(x_{0}\right)}{f}\left|D^{m} u-\left(D^{m} u\right)_{x_{0}, \rho}\right|^{p} \mathrm{~d} \mathcal{L}^{n} .
\end{aligned}
$$

Hence $f_{B_{\rho}\left(x_{0}\right)}\left|D^{m} u-\left(D^{m} u\right)_{x_{0}, \rho}\right|^{p} \mathrm{~d} \mathcal{L}^{n} \leqslant 1$ and

$$
\left(f_{B_{\rho}\left(x_{0}\right)}\left|D^{m} u-\left(D^{m} u\right)_{x_{0}, \rho}\right|^{p} \mathrm{~d} \mathcal{L}^{n}\right)^{1 / p}<\frac{S_{0}}{\sqrt{C_{p}^{2}+C_{p}^{p}\left(1+C_{p} \sqrt{n(n+2)}\right)^{p}}}
$$

imply $\Psi_{2}\left(x_{0}, \rho, P_{x_{0}, \rho}\right)<S_{0}$.

Since $y \mapsto\left(D^{m} u\right)_{y, \rho}$ and $y \mapsto f_{B_{\rho}(y)}\left|D^{m} u-\left(D^{m} u\right)_{y, \rho}\right|^{p} \mathrm{~d} \mathcal{L}^{n}$ are continuous, we see that (53) and (54) imply the same conditions near $x_{0}$. Now arguing as in the proof of Theorem 1 we get the desired partial regularity result. 


\section{REFERENCES}

[1] Acerbi E., Fusco N., Semicontinuity of problems in the calculus of variations, Arch. Rat. Mech. Anal. 86 (1984) 125-145.

[2] Ball J.M., Convexity conditions and existence theorems in nonlinear elasticity, Arch. Rat. Mech. Anal. 63 (1977) 337-403.

[3] Campanato S., Proprietà di una famiglia di spazi funzionali, Ann. Scoula Norm. Sup. Pisa 18 (1964) 137-160.

[4] Campanato S., Teoremi di interpolazione per tranformazioni che applicano $L^{p}$ in $C^{h, \alpha}$, Ann. Scoula Norm. Sup. Pisa 18 (1964) 345-360.

[5] Campanato S., Equazioni ellitichi del $\mathrm{II}^{e}$ ordine e spazi $\mathcal{L}^{2, \lambda}$, Ann. Mat. Pura Appl. 69 (1965) 321-381.

[6] Campanato S., Alcune osservazioni relative alle soluzioni di equazioni ellittiche di ordine 2m, in: Atti Convegno Equaz. Der. Parz., Bologna, 1967, pp. 17-25.

[7] Dacorogna B., Direct Methods in the Calculus of Variations, Springer, Berlin, 1989.

[8] Duzaar F., Steffen K., Optimal interior and boundary regularity for almost minimizers to elliptic variational integrals, preprint.

[9] Evans L.C., Quasiconvexitity and partial regularity in the calculus of variations, Arch. Rat. Mech. Anal. 95 (1986) 227-252.

[10] Evans L.C., Gariepy R.F., Measure Theory and Fine Properties of Functions, CRC Press, Boca Raton, 1992.

[11] Giaquinta M., Multiple Integrals in the Calculus of Variations and Nonlinear Elliptic Systems, Princeton University Press, Princeton, 1983.

[12] Giaquinta M., Introduction to Regularity Theory for Nonlinear Elliptic Systems, Birkhäuser, Basel, 1993.

[13] Giaquinta M., Modica G., Regularity results for some classes of higher order nonlinear elliptic systems, J. Reine Angew. Math. 311 (1979) 145-169.

[14] Giaquinta M., Modica G., Partial regularity of minimizers of quasiconvex integrals, Ann. Inst. Henri Poincaré, Analyse non Linéaire 3 (1986) 185-208.

[15] Guidorzi M., Poggiolini L., Lower semicontinuity of quasiconvex integrals of higher order, Nonlinear Differ. Equations Appl. 6 (1999) 227-246.

[16] Gilbarg D., Trudinger N.S., Elliptic Partial Differential Equations of Second Order, Springer, Berlin, 1983.

[17] Marcellini P., Approximation of quasiconvex functions and lower semicontinuity of multiple integrals, Manuscripta Math. 51 (1985) 1-28.

[18] Meyers N.G., Quasi-convexity and lower semi-continuity of multiple variational integrals of any order, Trans. Amer. Math. Soc. 119 (1965) 125-149.

[19] Morrey C.B., Quasiconvexity and the lower semicontinuity of multiple integrals, Pacific J. Math. 2 (1952) 25-53.

[20] Simon L., Lectures on Geometric Measure Theory, Australian National University Press, 1983.

[21] Simon L., Theorems on Regularity and Singularity of Energy Minimizing Maps, Birkhäuser, Basel, 1996.

[22] Zhou X.P., Weak lower semicontinuity of a functional with any order, J. Math. Anal. Appl. 221 (1998) 217-237. 